\title{
A review on wall slip in high solid dispersions
}

Michel Cloitre ${ }^{(1)}$ and Roger T. Bonnecaze ${ }^{(2)}$

${ }^{1}$ Soft Matter and Chemistry, CNRS, ESPCI Paris, PSL Research University, 10 Rue Vauquelin, 75005 Paris, France

${ }^{2}$ McKetta Department of Chemical Engineering and Texas Materials Institute, The University of Texas at Austin, Austin, TX 78712, USA

\begin{abstract}
High solid dispersions are soft materials made of colloidal or non colloidal particles dispersed at high volume fractions in a liquid matrix. They include hard sphere glasses, colloidal pastes, concentrated emulsions, foams, and vesicles. These materials are prone to exhibit different kinds of flow heterogeneities: shear-banding, wall slip, and fracture. While wall slip is often considered as a nuisance by experimentalists, it appears to be a fundamental component to the way that high solid dispersions respond to mechanical deformation. Moreover the ability of soft materials to slip onto surfaces allows them to move readily and efficiently in many natural phenomena and industrial processes. This review surveys recent developments and current research in the field. Topics like wall slip detection and control, microscopic modeling for rigid and soft particles materials, and the relation between wall slip and other flow heterogeneities are discussed. We also identify important open issues for future research.
\end{abstract}

\section{Submitted to Rheologica Acta \\ (Special Issue - Bingham Centennial Anniversary)}

November 2016 


\section{Introduction}

Many materials we encounter in real life are neither perfect elastic solids nor ordinary viscous or viscoelastic liquids. They appear to be able to sustain their own weight and maintain their shape under gravity, while deforming appreciably when subject to a large enough stress. A day-to-day illustration of this interesting behavior is toothpaste which spreads out only when the tube is strongly pressed. From a macroscopic point of view, the transition between liquidlike and solid-like properties occurs over a narrow range of stress values and is characterized by the so-called yield stress below which the material can be considered as a solid, albeit slowly evolving whereas above which it flows (Barnes 1999). This unique feature is exploited in many applications for instance to process high-performance coatings, solid inks, ceramic pastes, textured food and personal care products.

The rheological behavior of yield stress materials is often quantified by the Herschel-Bulkley equation, $\sigma=\sigma_{y}+k \dot{\gamma}^{n}$ or the Bingham equation when $n=1, \sigma_{y}$ being the yield stress, $k$ the consistency and $n$ the shear thinning exponent (Barnes 1999). This phenomenological description applies to very different materials even though the existence and physical meaning of yielding can have different origins: associative polymers (Tsitsilianis and Iliopoulos 2002), biopolymer gels (Whitcomb and Macosko 1978; Ross-Murphy 1995; Stokes and Frith 2008), textured phases of liquid crystals and block copolymers (Cloitre and Vlassopoulos 2011), and concentrated suspensions (Bonnecaze and Cloitre 2010). Given the broadness of the field, we restrict this review to high solid dispersions made of colloidal or non-colloidal particles dispersed at high volume fractions in a liquid matrix. A well-known example is a Brownian hard sphere glass, where particles experience excluded volume interactions only. In glasses $(0.58<\phi<0.64)$, the particles are temporarily trapped in cages which resist deformation entropically but break when a large enough stress exceeding their mechanical resistance is applied (Besseling et al 2009). The yield stress of entropic glasses is of the order of a fraction of Pascal, which precludes their use as thickeners in most applications. At the jamming transition $\left(\phi_{\mathrm{C}} \cong 0.64\right)$, the suspension is at the isostatic point and becomes marginally rigid with six contacts per particle in average (van Hecke 2010). When particles are soft and deformable, they can be packed much above the jamming transition (van Hecke 2010; Vlassopoulos and Cloitre 2014). In jammed suspensions, thermal motion plays no role even for otherwise Brownian particles and the yield stress is associated with the network of interparticle contacts. This explains why materials as different as microgel pastes (Seth et al 
2012), concentrated emulsions and foams (Cohen-Addad and Höhler 2014), multiarm star polymer solutions (Vlassopoulos and Fytas 2010), dense packings of multilamellar vesicles (Fujii and Richtering 2006) and many other materials share similar yielding properties. The existence of short-range attractive forces between particles brings additional complexity. Attractive interactions can lead to the formation of a space-spanning network or a gel, which is able to sustain a finite stress but rearranges and breaks above a large enough stress. In colloidal gels yielding is a complex process which is ultimately determined by the strength of the attractive interactions and the micromechanical rearrangements allowed by the network (Vermant and Solomon 2005). It has been proposed to classify yield stress materials into two distinct types: simple yield stress materials and thixotropic yield stress materials like colloidal gels, both categories exhibiting different flow properties (Bonn and Denn 2009; Ovarlez et al 2013). Yield stress materials have been the subject of several reviews to which we refer the reader interested in a deeper understanding of these materials (Chen et al 2010; Coussot 2014; Bonn et al 2015).

Yield stress materials are prone to exhibit different kinds of flow heterogeneities. Figure 1 presents schematics of velocity profiles in simple shear for homogeneous flows, shear-banded flows, slip flows, and apparent slip flows. In homogeneous flows, the velocity profiles vary linearly between the shearing surfaces and the resulting local shear rates are equal to the macroscopic shear rates (Fig. 1a). Shear banding denotes a broad class of phenomena of different origins, which are associated with the spatial localization of the strain or shear rate into one or several layers of finite thickness (Fig. 1b). Shear banding has been observed and described in materials as different as polymeric fluids, wormlike micelles, granular media, and thixotropic yield stress materials. The phenomenon has stimulated a lot of work and it is the subject of several reviews, some of them specifically dedicated to high solid dispersions (Goddard 2003; Olmsted 2008; Schall and van Hecke 2010; Fielding 2014; Divoux et al 2016). Note that fracture which is sometimes observed in dense suspensions can be viewed as a particular case of shear banding where the material loses its cohesion and breaks apart (Smith 2015). Slip represents an extreme realization of strain localization where most of the deformation occurs near the confining walls whereas the bulk of the materials behaves more or less like a solid body or exhibits negligible deformation. The generic origin of slip has been formalized by Bingham himself as "a lack of adhesion between the material and the shearing surface. The result is that there is a layer of liquid between the shearing surface and the main body of the suspension" (Bingham 1922). Nowadays it is usual to distinguish true slip, when 
the slip layer is of molecular dimension (Fig. 1c), from apparent slip when the local velocity varies over a finite, albeit small, mesoscopic distance (Fig. 1d) (Barnes 1995). True slip is relevant for polymers melts or solutions whereas slip of high solid dispersions is generally classified as apparent slip (Barnes 1999; Hatzikiriakos 2015).

The fact that high solid dispersions tend to slip at bounding surfaces rather than deform and flow has long be considered as a source of artifacts with respect to the rheological characterization of these materials. When wall slip is present, it causes important errors in the determination of the yield stress and other material properties. This has prompted the development of technical methods to correct data from the contribution of wall slip (Mooney 1931; Yoshimura and Prud'homme 1988). Another reason why wall slip is extremely important is that it generally occurs, and cannot be avoided, during the use and processing of dispersed materials. Thus the slip behavior of materials needs to be characterized and used as appropriate boundary conditions in the mathematical modeling and simulation of complex processes like capillary flows, slit flows and extrusion flows (Lawal and Kalyon 1994a, b; Kalyon et al 1999; Kalyon 2005). The existence of slip has also important ramifications on the development of flow instabilities during the extrusion of highly filled polymeric suspensions (Denn 2001; Birinci and Kalyon 2006; Tang and Kalyon 2008), the spreading of yield stress fluids (Luu and Forterre 2009; Saïdi et al 2011, Jalaal et al 2015), and the establishment of steady conditions during start up flows or cessation flows of yield stress materials (Damaniou et al 2014; Philippou et al 2016). Far from being just a nuisance, slip is thus fundamental to the way that high solid dispersions respond and behave.

There are also many situations where slip is desirable because it is precisely the ability of soft materials to slip that allows them to move readily and efficiently. Slip is essential within many natural and biological processes, including transport of solid foods during the oral, digestion and waste pathways (Stokes et al 2013), the movement of red blood cells through narrow arteries (Roman et al 2016), the adhesive locomotion of gastropods (Ewoldt et al 2007), or the nutrient delivery by cytoplasmic streaming in plant cells (Wolff et al 2013). The presence or absence of slip also contributes to the sensory perception of emulsion-based personal care products and cosmetics (Ozkan et al 2011). Finally, slip participates to the transport of many complex suspensions, such as mineral suspensions, paints, foods, pharmaceuticals, sewage treatment and soils. For instance, slip of concentrated emulsions is at the origin of water-lubricated transport of heavy viscous oil or coal (Joseph 1997; Chen et al 2009). In oil pipelining a thin water film or light hydrocarbon is injected around the internal 
oil core, which, in the so-called core-annular flow regime, leads to efficient lubrication of the core flow (Joseph et al 1997). In the context of confined flows and microfluidic devices where surface effects dominate, slip can be exploited to manipulate the transport of complex fluids by changing the surface topography, the physical roughness and the chemical composition of the boundaries. A remarkable example is the control of ideal plug flows inside the dispensing nozzles of colloidal inks in 3D printing systems or during screen printing of silver pastes for silicon solar cells (Smay et al. 2002; Xu et al. 2017).

The ubiquity of wall slip in soft matter science as well as its practical and technological importance has motivated a great interest from physicists and engineers. Several reviews describing general properties of slip in polymeric and colloidal materials are already available (Barnes 1995; Granick et al 2003; Lauga et al 2007; Sochi 2011; Hatzikiriakos 2015). Since the well-known review by Barnes in 1995, which pioneered the field, more than 1600 articles mentioning 'wall slip' in the title have been published. This profusion of studies concerns many different materials and flows making the topic extremely rich but somewhat confusing. Despite this large body of research, it remains challenging to get microscopic insight into slip phenomena and understand their dependence on surface characteristic, flow rate, and material properties. A new review along this direction is thus timely and highly desirable.

\section{Detection of wall slip}

\subsection{Macroscopic methods based on rheology}

When slip occurs, the overall deformation of the material is localized in a thin layer of thickness $\delta$ adjacent to the confining walls where most of the dissipation takes place, resulting in a large velocity gradient at the wall (Fig 1d). Hence the actual deformation and deformation rate experienced by the material is drastically different from the effective shear rate which is applied or measured. An immediate consequence discussed in many textbooks is that the apparent flow properties depend on the characteristic size of the flow with respect to $\delta$ in the presence of slip. Indeed, experimentally it is observed that the apparent shear viscosities measured in capillaries with the same length-to-diameter ratios but different diameters do not coincide. Similarly, rheological properties obtained in Couette flows depend on the gap values.

Mooney (1931) introduced a method to extract slip velocities from multiple measurements performed in capillary rheometry. Capillaries with the same length over diameter ratios but different diameters $D$ are used. The analysis is based on the following equation that states that 
plots of the apparent shear rate $\dot{\gamma}_{a}=32 Q / \pi D^{3}$ ( $Q$ : flow rate) versus the inverse diameter at constant pressure should give a straight line with a slope proportional to the slip velocity $V_{\mathrm{S}}$ :

$$
V_{S}=\left.\frac{1}{8} \frac{\partial \dot{\gamma}_{a}}{\partial(1 / D)}\right|_{\Delta P}
$$

The method was later revisited by several authors (Yoshimura and Prud'homme 1988; ; Yilmazer and Kalyon 1989; Wien and Tovchigrechko 1992). In particular Yoshimura and Prud'homme (1988) proposed a new analysis for Couette and parallel plate viscometers, which uses only two measurements. The method requires the assumption that the slip velocity depends on the wall shear stress $\sigma_{\mathrm{W}}$ only, not on the gap $h$ between the shearing surfaces or other components of the stress tensor. The apparent shear rate, $\dot{\gamma}_{a}$, has a simple expression as a function of the true shear rate, $\dot{\gamma}$, the slip velocity $V_{\mathrm{S}}$, and the gap $h: \dot{\gamma}_{a}=\dot{\gamma}+2 V_{S} / h$. From two experiments performed at the same stress $\sigma_{\mathrm{W}}$ at two different gap values $h_{1}$ and $h_{2}$, it is thus possible to deduce the slip velocity from $\dot{\gamma}_{a}^{(1)}$ and $\dot{\gamma}_{a}^{(2)}$ :

$$
V_{S}\left(\sigma_{W}\right)=\frac{\dot{\gamma}_{a}^{(1)}\left(\sigma_{W}\right)-\dot{\gamma}_{a}^{(2)}\left(\sigma_{W}\right)}{1 / h_{1}-1 / h_{2}} \text {. }
$$

The knowledge of the slip velocity gives access to the true shear rate experienced by the material. This method has been applied by many authors to very different materials. Yeow et al analyzed the conversion of capillary viscometry data with wall slip into a shear stress versus shear rate relationship and a wall shear stress versus slip velocity relationship as an illposed inversed problem and solved it using a numerical procedure based on Tikhonov regularization (Yeow et al 2003; Kalyon 2003; Zahirovic et al 2009).

Large Amplitude Oscillatory Shear (LAOS) can be used as a substitute of steady shear flow to probe the rheology of high solid dispersions (Hyun et al 2011). An interesting question concerns the detection of slip in materials subjected to LAOS in rotational rheology. Slip sometimes introduces a dissymmetry in the stress wave form between the positive and negative parts of the oscillations (Hatzikiriakos and Dealy 1991). Fourier-transform rheology offers a convenient way of analyzing LAOS signals by decomposing the stress responses in the time domain into a series of harmonics in the frequency domain (Wilhem et al 1998). Several authors have proposed to relate the presence of even harmonics to wall slip, without any further measurements at more than one gap or on both slip and non-slip surfaces (Graham 1995; Reimers and Dealy 1996; Ozkan et al 2012). Unfortunately wall slip can take place 
without the occurrence of a dissymmetry in the wave form and thus the appearance of even harmonics in the stress response when the material slips and sticks at symmetric locations of the cycles. In addition, wall slip is not a necessary condition (Atalika and Keunings 2004). Dissymmetric waveforms and even harmonics can be caused by other flow heterogeneities like imperfect alignment of the upper and lower plates of the rheometer, shear-banding, elastic instabilities, or fluid ejection. A recent interpretation of Fourier-Transform rheology models the whole spectrum as a superposition of spectra associated with various nonlinear phenomena including wall slip (Klein et al 2007).

To conclude, the methods based on rheology alone implicitly assume that slip is the only source of disturbance responsible for a dependence of the flow properties on the size. This limitation has prompted the development of techniques allowing for the qualitative visualization of velocity fields or the direct measurement of local velocities and velocity profiles in steady or unsteady conditions. It is interesting to note that these techniques are not restricted to the study of wall slip and have proven to be extremely useful to investigate other kinds of flow localization like shear-banding, fracture, or elastic instabilities (Manneville 2008).

\subsection{Microscopic techniques}

\section{Visualization of wall slip}

The first direct attempts to visualize wall slip in a yield stress undergoing steady shearing motion between two parallel plates consisted in examining the distortion a vertical line perpendicular to the velocity field during flow (Magnin and Piau 1987, 1990; Kalyon et al 1993). Before applying the deformation, a straight line marker is placed at the free surface of the suspension and the edges of the shearing plates. Upon deformation, discontinuities appear at both suspension/wall interfaces, revealing wall slip. The relative displacement of the line between the suspension and the plate gives an estimate of the wall velocity. The method is purely qualitative and suffers from poor accuracy at the wall-suspension interface where slip takes place. In addition, in the presence of slip, the flow at the edge of the geometries does not always match the bulk flow structure (Meeker et al 2004b).

\section{Laser Doppler velocimetry}

Several methods based on laser Doppler velocimetry (LDV) have been developed and used to measure velocity profiles. Jana et al (1995) were probably the first to implement LDV to measure wall slip velocity in concentrated suspensions. They used a conventional method 
which consists in illuminating the sample with two laser beams that intersect at a point and create a fringe pattern. Particles passing through the pattern scatter a signal which is processed to yield the local velocity. Later on Salmon et al (2003a) implemented a variant, known as heterodyne Dynamic Light Scattering, where the Doppler-shifted scattered light that has crossed the flow is mixed to a reference beam, which produces interferences leading to a signal oscillating in time at a frequency $\mathbf{q v}$ ( $\mathbf{q}$ is the scattering wave vector; $\mathbf{v}$ is the velocity vector). The spatial resolution is in the range $50-100 \mu \mathrm{m}$ and it takes about 10 to $100 \mathrm{~s}$ to record a full velocity profile. LDV has been used to study flow properties of yield stress materials as different as concentrated emulsions (Salmon et al 2003b), wormlike micelles (Salmon et al 2003b), Laponite suspensions (Ianni et al 2007), and rod-like particle glasses (Dhont et al 2017).

\section{Digital image methods}

Digital image methods refer to techniques based on the imaging at high magnification of fluorescent micron-size particles within a flowing liquid. The particles are transported by the fluid, and the determination of their displacements allows for the measurement of local velocity profiles. Digital image methods can be used in parallel to macroscopic measurements by coupling a conventional or confocal microscope to a commercial rheometer (Cohen et al 2006; Besseling et al 2009; Jop et al 2012). They are widely implemented in microfluidic setups to measure confined flow profiles in microchannels (Isa et al 2007; Li et al 2014).

To measure particle displacements, different digital particle analyses are available. Micro Particle Image Velocimetry ( $\mu$ PIV) evaluates the local displacements of particles from the correlation between consecutive pairs of images. The displacements are given by the position of the maximum of intensity of the pair correlation. The rate of acquisition of velocity profiles is variable depending on the performance of the equipment and the geometry of detection; it is typically of a few seconds but rates as low as a few milliseconds have been reported (Miller and Rothstein 2007). The spatial resolution is currently of the order of $10 \mu \mathrm{m}$ but it can be decreased to about $1 \mu \mathrm{m}$ in some cases ( $\mathrm{Li}$ et al 2014). $\mu$ PIV has been used to characterize wall slip properties of yield stress materials as different as microgel suspensions (Vayssade et al 2014), concentrated emulsions (Jop et al 2012; Paredes et al 2015), wet foams (Blondin and Doubliez 2002; Le Merrer et al 2015), gel particle suspensions (Pérez-González et al 2012; Aktas et al 2014; Poumaere et al 2014; Maillard et al 2015; Ahongio et al 2016; Ortega-Avila et al 2016), non-Brownian hard sphere suspensions (Jesinghausen et al 2016). 
Another established method is Particle Tracking Velocimetry (PTV). Here individual tracers are tracked between two consecutive images. The positions of particles are detected on each image by their maximum intensity or the intercorrelation with the theoretical point spread (Crocker and Grier 1996). It is easy to compute the displacement and the velocity of each particle from its coordinates. The spatial resolution is comparable to that of the $\mu$ PIV method. This method is widely implemented and has been used to measure the yield velocity of microgel suspensions (Meeker et al 2004b; Seth et al 2012); entangled polymer solutions (Boukany et al 2010), hard sphere suspensions (Isa et al 2007; Ballesta et al 2008, 2012; Ghosh et al 2016), gel particle suspensions (Geraud et al 2013).

\section{Ultrasonic velocimetry}

Ultrasound is a nonintrusive probe that can be applied to soft materials which are not optically transparent. Ultrasonic Speckle Velocimetry (USV) is based on the Doppler effect: a short high frequency ultrasonic pulse is sent to the fluid periodically and the echoes issuing from the particles suspended in the fluid are collected. A dedicated analysis of the signal and a calibration step accounting for the geometry provide the position and the velocity of the particles (Manneville et al 2004; Gallot et al 2012). The technique has been implemented on commercial rheometers equipped with Couette geometries. It allows for the determination of full velocity profiles with a spatial resolution of about $40 \mu \mathrm{m}$ but a very high temporal resolution (0.02-2 s), which makes the technique unique to resolve transient or non-stationary phenomena like stick-slip. USV is successfully used to study a variety of yield stress materials exhibiting a complex flow structure combining for instance shear banding and wall slip: concentrated emulsions (Bécu et al 2006), block copolymer micelles (Manneville et al 2007), Laponite suspensions (Gibaud et al 2008, 2009), carbon black gels (Gibaud et al 2010), gel particle suspensions (Divoux et al 2011a, b; Divoux et al 2012), fiber suspensions (Derakhshandeh et al 2012).

\section{Magnetic Resonance Imaging}

Flow Magnetic Resonance Imaging (MRI) consists in imaging sequences of the fluid in a way that the pixels of the images are encoded with a measure of the molecular displacement over time. A detailed description of the technique exceeds the scope of this review and we refer the interested reader to reference articles in the field (Callaghan 1999, 2006; Gladden and Sederman 2013). Flow MRI has two main advantages; it does not have transparency limitations like optical methods for instance, and three dimensional velocity fields can be determined in a variety of complex geometries including laboratory extruders (Amin et al 
2003; Barnes et al 2006; Rabideau et al 2010). However, there are several disadvantages such as the cost of the equipment and the difficulty to adapt a MRI setup on commercial rheometers to obtain simultaneous measurements of global stress and local velocity, due to the presence of strong magnetic field. The spatial resolution is of the order of $50 \mu \mathrm{m}$ and the time to record a full velocity profiles is generally of the order of $10 \mathrm{~s}$. MRI velocimetry has been used to study the flow behavior of solutions and suspensions of many complex fluids (Callaghan 2008; Manneville 2008). Flow MRI has proven a decisive tool to detect and characterize the phenomenology of shear localization in high solid dispersions such as concentrated star solutions (Holmes et al 2004), thixotropic bentonite suspensions (Raynaud et al 2002), drilling muds (Ragouilliaux et al 2006), cement pastes (Jarny et al 2005), gel particle suspensions (Coussot et al 2009; Ovarlez et al 2013). Flow MRI has been applied to identify and study slip phenomena in polymer solutions (Rofe et al 1996), colloidal hard sphere suspensions (Wassenius and Callaghan 2005), polymer and surfactant solutions (Gibbs et al 1996; Mair and Callaghan 1997), gel particle suspensions (Gibbs et al 1996), emulsions (Bertola et al 2003; Hollingsworth and Johns 2006), and sewage wastes (Tabuteau et al 2004).

\section{Near-field optical methods}

The spatial resolution of most of the techniques described above is not sufficient to resolve the interior of the lubricating films involved in slip phenomena. This has prompted the development of several near-field techniques which are able to probe wall-material interfaces at scales of a few tens of nanometers.

The general principle is to probe the wall-fluid interface with an evanescent light field. Different variants have been explored. Hartman Kok et al $(2001,2004)$ used attenuated total reflection infrared spectroscopy (ATR-IR) to measure concentration profiles of Brownian particles near solid walls at volume fractions up to $\phi=0.3$. The local velocity of liquids near solid walls has been measured with resolution of about $50 \mathrm{~nm}$, and slip detected using a combination of internal reflection and fluorescence recovery after photo-bleaching (TIRFRAP) (Pit et al 1999; Schmatko et al 2005). Recently, a new near-wall velocimetry technique, based on evanescent wave dynamic light scattering, has been described (Loppinet et al 2012). It allows for the measurement of near-wall velocity profile with a resolution of tens of nanometers. 
Particle Tracking Velocimetry (PTV) has been extended to measurements at a submicron resolution using Total Internal Reflection Fluorescence (TIRF) with the objective to investigate the close vicinity of surfaces where slip takes place. Similarly to $\mu$ PTV, the nanoPTV-TIRF technique consists of seeding the fluid with fluorescent nanoparticles that are illuminated by an evanescent field near a wall-fluid interface (Huang et al 2006; Bouzigues et al 2008; Li et al 2015). The intensity of the light which is emitted by the particles decreases exponentially with the distance to the surface, providing a way to determine their spatial position with respect to the wall. The resolution can be as low as $30 \mathrm{~nm}$, the time resolution being limited by the scanning performance of the opto-mechanical setup and the acquisition rate of the camera. Although the technique appears to be very promising to investigate lubricating films in concentrated emulsions, foams, polymer solutions, colloidal glasses, jammed microgel suspensions, it is relatively delicate to implement and may be subject to artifacts, which explain why it has not yet been used to a large extent.

\subsection{Generic signatures of wall slip}

Slip of high solid dispersions exhibits generic signatures irrespectively of the composition of the dispersions and the nature of the particles. In this section we examine the slip behavior of concentrated dispersions in rheometric flows and pressure driven flows.

\section{Rheometric flows}

Due to their high effective viscosity, yield stress materials are preferentially investigated in parallel plates or cone and plate geometries working in controlled stress or controlled strain modes. Figure 2a shows that typical flow curves of a microgel suspension measured using both rough and smooth surfaces. When sheared using rough surfaces, high solid dispersions have the characteristic behavior expected for a yield stress fluid with a flow curve described by the Herschel-Bulkley equation (Seth et al 2011). When the surfaces are smooth, slip is possible and the behavior is dramatically changed. At high shear rates the flow curve coincides with that obtained with rough surfaces. However, the measured shear rate no longer tends to zero at the bulk yield stress $\sigma_{\mathrm{y}}$. Instead the flow curve display a kink at a shear rate $\dot{\gamma}_{a}^{*}$ and apparent flow continues to be detected for stresses well below $\sigma_{\mathrm{y}}$. Flow curves below the yield stress are very sensitive to the chemical nature of the shearing surfaces (Seth et al 2008, 2012). At very low stresses the flow curve sometimes exhibits an apparent yield stress also termed slip yield stress, which is interpreted as the lowest stress above which steady slip motion is possible. The slip yield stress should not be confused with the bulk yield stress. This 
behavior is generic and has been observed in a variety of materials including microgel suspensions (Meeker et al 2004a, 2004b ; Seth et al 2008, 2012), gel particle suspensions (Piau 2007; Métivier et al 2012), concentrated emulsions (Princen 1985; Bower et al 1999; Bertola et al 2003; Salmon et al 2003b; Egger and McGrath 2006; Seth et al 2012; Paredes et al 2015; Habibi et al 2016), foams (Marze et al 2008), hard sphere glasses (Ballesta et al 2008; Ballesta et al 2012), colloidal gels (Buscall et al 1993, 2010; Walls et al 2003; Ballesta et al 2013; Grenard et al 2014), concentrated non-Brownian suspensions (Kalyon et al 1993), biosolids (Tabuteau et al 2004). It clearly distinguishes concentrated suspensions, where slip occurs at low shear rates where they are solid-like, from polymeric materials where slip takes place at large velocities. An interesting remark concerns the squeeze flow of soft solids, where the signature of slip is also a deviation of the squeezing force at low squeezing velocities (Lawal and Kalyon 1998; Meeten 2004a).

Direct in-situ measurements of the velocity profiles using one of the techniques discussed in Section 1.2 provides a physical interpretation of the flow curves in the presence of slip. This is illustrated in Fig. $2 b$ which represents the velocity profiles of the same microgel suspension as in Fig 1a, which have been measured using Particle Tracking Velocimetry. At very low applied velocities corresponding to stresses below the yield stress, the material simply slips as a bulk solid on the shearing surfaces: this is the regime of full slip where apparent motion is only due to wall slip. Just above the yield point, the material keeps on slipping on the surfaces but the material starts to yield; however, wall slip still dominates the overall motion and yielding just brings a small additional contribution. At higher shear rates, wall slip becomes negligible with respect to the deformation of the material which yields. This leads us to distinguish three flow regimes: full slip below the yield stress, full yielding well above the yield stress and a transition regime in between where slip combines with yielding (Bertola et al 2003; Meeker et al 2004a, b). The transition region having a limited extension, it can sometimes be assimilated to the full yielding region (Ballesta et al 2008, 2012).

In most experiments, the slip layer is not spatially resolved and the slip velocity is inferred from the intersection of the velocity profiles with the shearing surface. For stresses at and below the paste yield stress, it is also possible to obtain a good measure of the slip velocity $V_{\mathrm{S}}$ from rheology data only. Indeed, since all the motion comes from the slipping of the paste, the slip velocity $V_{\mathrm{S}}$ is simply the cone or plate velocity $V_{0}$ for slip at one wall or $V_{0} / 2$ for slip at both walls (Meeker et al 2004b). Since in this regime, the slip velocity is directly related to the local velocity imposed by the cone of plate geometry whereas yielding depends on the 
stress, slip gets more pronounced for smaller radii whereas yielding eventually dominates at the periphery. This demonstrates that, in presence of slip, the stress in a cone-plate, while essentially uniform across the gap, can be radially non-uniform (Meeker et al 2004b; Ballesta et al 2012). This is obviously true in the transition regime but also in the full slip regime where, in addition, the nominal stress given by the rheometer differs from the mean stress (Meeker et al 2004b; Ballesta et al 2012).

The generic response of yield stress materials subject to LAOS in the presence of wall slip can be mapped on the steady shear situation (Shewan 2017). A useful criterion is to compare the yield stress $\sigma_{\mathrm{y}}$, the stress which limits the linear regime $\sigma_{0}$, and the slip yield stress $\sigma_{\mathrm{S}}$. When $\sigma_{0}<\sigma_{\mathrm{S}}<\sigma_{\mathrm{y}}$, slip does not affect the linear response so that the storage and loss moduli can be reliably measured. Otherwise strong deviations of the linear viscoelastic moduli from their actual values are observed. At large applied strains or stresses, the material is entirely fluidized and the effect of slip is negligible. In between these two regimes, slip affects the LAOS response (Walls et al 2003).

\section{Pressure driven flows}

Pressure driven flows of high solid dispersions in microchannels, channels or other complex geometries like slit or extrusion dies are particularly relevant to the industrial processing of pastes, highly filled composites and ceramics (Lawal and Kalyon 1994b; Wilson and Rough 2006; Kalyon 2010; Westenberg et al 2010). This type of flows has thus attracted a lot of attention and again it is possible to highlight several common features shared by different yield stress materials (Kalyon 2005; Pérez-González et al 2012; Aktas et al 2014; Poumaere et al 2014; Vayssade et al 2014; Ortega Avila et al 2016).

Figure 3a represents normalized velocity profiles of a concentrated gel particle suspension in capillary flow measured by PIV (Aktas et al 2014). The velocity profiles have the characteristic shape expected for yield stress materials: they exhibit a central unyielded region termed plug flow and two sheared zones adjacent to the walls. When the pressure gradient is low, the wall shear stress is smaller than the yield stress, plug flow spans the entire capillary and the motion of the suspension is due to wall slip only. This regime is equivalent to the full slip situation in rheometric flows. At higher pressure gradient, yielding occurs, the radius of the unyielded region decreases with increasing pressure gradient whereas the shear zones increases. As before the slip velocity can be measured by extrapolating the velocity profiles to the capillary walls. The importance of wall slip with respect to yielding can be quantified by 
plotting the ratio of the volumetric flow rate due to slip over the volumetric flow rate (for capillary flow), or the ratio of the slip velocity over the mean velocity (rheometric flow). The data for the experiment of Fig. 3a are shown in Fig. 3b. At low pressure gradients corresponding to stresses below the yield stress, the ratios $Q_{\mathrm{S}} / Q$ and $V / V_{\mathrm{m}}$ are equal to 1 as expected. The stress at which both quantities deviate from 1 marks the onset of yielding which provides an alternative way to determine the yield stress (Aktas et al 2014).

\section{Slip boundary conditions}

From a quantitative perspective the slip properties of high solid dispersions are often expressed in terms of a boundary equation relating the slip velocity to the wall shear stress. Meeker et al (2004a,b) proposed the general form valid below the yield stress, which accounts for a finite slip yield stress:

$$
V_{S}=V^{*}\left(\frac{\sigma_{W}-\sigma_{S}}{\sigma_{y}-\sigma_{S}}\right)^{m},
$$

where $\sigma_{W}$ is the wall shear stress, $\sigma_{\mathrm{S}}$ the slip yield stress, $m$ the slip exponent and $V^{*}$ the slip velocity at the yield stress $\sigma_{\mathrm{y}}$. The characteristic velocity $V^{*}$ was determined from the apparent shear rate $\dot{\gamma}_{a}^{*}$ which marks the onset of full slip using $V^{*}=\dot{\gamma}_{a}^{*} h$, where $h$ is the gap at the edge of the geometry (Meeker et al 2004b). It depends on material properties. Above the yield stress Divoux et al (2015) used the form:

$$
V_{S}=B\left(\sigma_{W}-\sigma_{y}\right)^{m},
$$

where $m$ is again the slip exponent and $B$ a prefactor. When $\sigma_{\mathrm{S}}<<\sigma_{\mathrm{W}}<\sigma_{y}$ and $\sigma_{\mathrm{W}}>>\sigma_{y}$, expressions (3) and (4), respectively, reduce to a form proposed by Kalyon (2005):

$$
V_{S}=\beta \sigma_{W}^{m}
$$

$\beta$ is the slip coefficient. When the slip exponent $m$ is equal to 1 , the classical Navier slip condition is recovered (Navier 1827). Note that the boundary conditions (3)-(5) are sometimes expressed in the form $\sigma_{W}=f\left(V_{S}\right)$. In general the parameters $\sigma_{S}, \beta$, and $m$ depend on the particle properties, the solvent viscosity and the nature of the shearing surfaces. In the following, we review the state of the art for the cases of hard and soft particle concentrated suspensions respectively. An outstanding challenge is to express these parameters in terms of microscopic models, which requires to identify the physical mechanisms at the origin of slip. This will be our objective in the following Sections. While the specific behavior of slipping suspensions is associated with apparent slip, in most experiments the slip layer is too thin to 
be directly visualized. In the regime of full slip, an effective slip layer thickness can be estimated from:

$$
\delta=\frac{\eta V_{S}}{\sigma_{W}}
$$

where $\eta$ is the viscosity of the suspending medium. This relation is general and applies even if the viscosity is non-Newtonian (Kalyon 2005). With these notations, we have $\delta \cong \eta \beta$. Relations (3)-(6) will be central to our discussion in the following sections because they allow us to rationalize and compare the behavior of a broad range of materials in terms of the relationship between the slip velocity and the wall shear stress.

\section{Slip phenomena in rigid particle dispersions}

Wall slip is ubiquitous in rigid particle dispersions. As in other materials, slip is associated with the existence near the shearing walls of a thin layer from which particles are excluded, resulting in a low viscosity lubricating layer where the overall deformation is localized. Important questions concern the physical origin of the depletion layer, its properties (thickness, particle concentration), and its connection with macroscopic properties. Different mechanisms have been proposed to explain the formation of depleted layers in Brownian or non-Brownian rigid particle suspensions: (i) steric depletion: since the particles cannot penetrate the wall, the volume fraction at distances of the order of a particle radius to the wall is lower than that in the bulk (Yilmazer and Kalyon 1989; Kalyon 2005; Ballesta et al 2008, 2012); (ii) particle migration driven by gradients in shear rate in Couette geometries and channel flows or a non-equilibrium particle pressure (Leighton and Acrivos 1987; Jana et al 1995; Franck et al 2003; Semwogere et al 2007; Besseling et al 2010); (iii) other mechanisms such as flow-induced layering at surfaces or repulsive wall-particle forces (Cohen et al 2006; Korhonen 2015).

\subsection{Non yielding particle suspensions}

Particle suspensions can experience wall slip even though they do not have a yield stress. Hartman Kok et al $(2001,2004)$ observed wall slip in Brownian particle suspensions at volume fractions up to $\phi=0.3$. They used attenuated total reflection infrared spectroscopy (ATR-IR) to measure the concentration profiles of Brownian particles near the solid walls for Peclet numbers ranging from 0.01 to $45\left(P e=\tau_{B} \dot{\gamma}\right.$ where $\tau_{B}$ is a Brownian characteristic time and $\dot{\gamma}$ is the shear rate). They found that slip is negligible for $\mathrm{Pe}<1$ but that for $\mathrm{Pe}>1$ there exist a thin region where the particle concentration is lower than in the bulk. The 
thickness of this depleted layer is of the order of a few particles diameter and is in good agreement with effective slip layer thickness estimated from rheological measurements. Ghosh et al investigated the flow in microchannels of Brownian suspensions at volume fractions between 0.03 and 0.42 for Peclet numbers in the range 2-50, using confocal microscopy (Ghosh et al 2016). Particle wall depletion was observed over distances comparable to a particle diameter and was interpreted in terms of excluded volume. A simple model accounting for the decrease of viscosity in this boundary layer was found to match the experimental data. Non-Brownian suspensions at relatively low volume fractions $(\phi>0.2)$ also exhibit significant wall slip in Couette flows or pressure driven flows (Jana et al 1995; Ahuja and Singh 2009; Jesinghausen et al 2016). In Jana et al (1995) shear induced diffusion was considered to be responsible for particle wall depletion and slip.

\subsection{Brownian hard sphere suspensions and glasses}

Concentrated hard-sphere colloids constitute one of the simplest yield-stress fluids and a model for colloidal crystals and glasses. By imaging the deformation profiles of a colloidal crystal under oscillatory shear in confocal microscopy, Cohen et al (2006) showed that the flow of colloidal crystals exhibits a rich phenomenology including wall slip, yielding, and structural shear banding.

Glasses of polydisperse hard sphere exhibit significant wall slip. Ballesta et al (2008, 2012) performed a detailed study of wall slip in hard sphere glasses. The suspensions consisted of Brownian polymethacrylate colloids sterically stabilized in the glassy regime. They were studied using conventional rheology coupled to confocal microscopy. The suspensions obeyed the general phenomenology discussed in Section 2.3. In particular, the flow curves exhibited a characteristic kink which separates a low shear rate regime where the suspensions slipped as solids on the walls (full slip) and a high shear rate regime where the suspensions yielded and slip progressively became negligible compared to the bulk flow. In the full slip regime, the stress was expressed as an affine function of the slip velocity:

$$
\sigma=\sigma_{S}+\beta V_{S}
$$

which is a particular form of Eq 3 with $m=1$. The slip coefficient $\beta$ can be understood in terms of lubrication between the first layers of particles and the wall, with an effective lubrication layer thickness independent of the applied velocity. The stress $\sigma_{S}$ is the threshold slip stress which represents the minimum stress above which the particle distribution function reorganizes when slip sets in. Interestingly scaling $\beta$ with $\eta / R$ and $\sigma_{S}$ with $k T / R^{3}$ collapses the 
data obtained for different particle sizes and different viscosities when they are plotted as a function of 1- $\phi / \phi_{C}$ where $\phi_{C}$ is the random close packing (Fig. 4). The fact that $\beta$ and $\sigma_{S}$ are functions of $1-\phi / \phi_{C}$ indicates that steric depletion is the dominant mechanism at the origin of slip. Attempts to predict the slip coefficient $\beta$ by integrating the hydrodynamic friction at the wall using different forms of the pair distribution function are in qualitative agreement with the experimental data (Fig. 4a). The value of $\sigma_{S}$ is sensitive to van der Waal interactions but, surprisingly, is not related to some particle-wall Coulombic friction. Finally parameters $\beta, \sigma_{S}$, and the effective lubrication layer thickness $\delta$ are well described by semi-empirical expressions which suggest that $\beta$ and $\delta$ are connected to the osmotic pressure of the glasses (Fig. 4a) and $\sigma_{S}$ to the bulk yield stress (Fig. 4b). Although these results provide important microscopic insights about wall slip of colloidal glasses and establish a link with the physics of colloidal glasses, further work would be useful to get quantitative predictions for $\beta$ and $\sigma_{S}$.

\subsection{Concentrated non-Brownian hard sphere suspensions}

The question of slip in non-Brownian hard particle suspensions has attracted a lot of attention mainly because of the outstanding industrial relevance of these materials. Over the last 20 years, Kalyon and his coworkers have pioneered the field of highly filled suspensions, close to their maximum packing fraction, in relation with their industrial processing. They studied the flow and slip behavior of viscoplastic Herschel-Bulkley materials in parallel plate and capillary rheometers (Yilmazer and Kalyon 1989; Kalyon et al 1993; Aral and Kalyon 1994), single screw extrusion (Lawal and Kalyon 1994a, b), rectangular slit flows (Kalyon 2005), squeeze flow rheometers (Lawal and Kalyon 1998; Tang and Kalyon 2004). Kalyon proposed a phenomenological model based on the following hypotheses: (i) the slip layer consists only of the binder of the suspensions which sticks to the wall; (ii) its thickness is determined by the properties of the suspension only and is independent of the gap and flow rate (Kalyon 2005). The boundary condition in the presence of slip was expressed in the form of the generalized Navier expression (5) relating the wall shear stress, $\sigma_{\mathrm{w}}$, to the slip velocity, $V_{\mathrm{S}}$. When the suspending fluid is Newtonian, the slip exponent is $m=1$ and the slip coefficient is constant since $\beta=\delta / \eta$. For a shear thinning fluid characterized by a power law variation of the viscosity, $\eta=\eta_{0} \dot{\gamma}^{n}$, the slip exponent is $m=1 /(n+1)$ and the slip coefficient varies: $\beta=\delta / \eta_{0}^{1 /(n+1)}$. These boundary conditions have been implemented in analytical or simulation models for the extrusion of viscoplastic materials in the presence of wall slip (Kalyon 2010). Several other studies of highly filled suspensions deserve attention (Soltani 
and Yilmazer 1998; Gulmus and Yilmazer 2005; Gulmus and Yilmazer 2007; Lam et al 2007).

Let us turn our attention towards the origin of the slip layer in these concentrated nonBrownian suspensions. Yilmazer and Kalyon (1989) found that the ratio of the slip layer thickness over the particle diameter is of the order of 0.06 for $\phi=0.60$. Jana et al (1995) found a value of 0.063 for volume fractions in the range $0.46<\phi<0.52$. Soltani and Yilmazer (1998) determined values ranging from 0.04 to 0.07 . Kalyon (2005) proposed a correlation of the form $\delta / 2 R=1-\phi / \phi_{\mathrm{C}}$ where $\phi_{\mathrm{C}}$ is the maximum packing of rigid particles, which provides a good description of the experimental data available for multiple systems studied in different flow conditions. The fact that this correlation involves only geometric factors, namely the diameter of the particles and the volume fraction strongly suggests that steric depletion is the leading mechanism and particle migration does not an important role in these experiments.

\subsection{Colloidal gels}

Because of the presence of attractive interactions between the particles (silica, Laponite, clays, calcium carbonate) colloidal gels are generally thixotropic materials. The question of particle-wall interactions is also crucial because the presence of friction between the gels and the walls can affect the batch sedimentation of strongly flocculated colloids and thus the long term stability of formulations (Allain et al 1995; Condre et al 2006, Lester et al 2014). The behavior of colloidal gels exhibits several original features in comparison with that of well dispersed suspensions (Buscall et al 2010). First slip was shown to be a major complication which was particularly difficult to be obviated. For instance, Russell and Grant (2000) discovered that the strength of the particle-particle and particle-wall interactions can induce different behaviors. Depletion flocculated dispersions formed weak gels with good adhesion at the wall, which were not slipping. Stronger gels were shown to slip, which profoundly affected the rheological response. The importance of the interactions was also discussed by Walls et al (2003). A generic feature of colloidal gels is the difficulty to suppress wall slip with the use of rough surfaces (Grenard et al 2014; Ballesta et al 2013). Specific surfaces with roughness comparable to the largest aggregates in the gels are necessary to efficiently prevent gels from slipping (Ballesta et al 2013). Buscall et al (1993) proposes to analyze the rheological response of colloidal by systematically comparing data obtained with and without slip. Ballesta et al (2013) describes slip of colloidal gels as a surface yielding phenomenon 
related to the restructuring of clusters with time. A dynamic phase diagram featuring the dependence of slip on interaction strength and colloid volume fraction was built.

\section{Slip phenomena in soft particles dispersions}

Soft and deformable particles form a broad class of industrially relevant materials which share common features: microgels and particle gels, emulsions, vesicles, and foams (Vlassopoulos and Cloitre 2014). They are extremely sensitive to the wall-slip phenomenon because they deform at solid contacts and are able to bypass wall irregularities or corrugations introduced to suppress wall slip (see Section 6). In this section we review the state of the art for microgel and gel particle suspensions, concentrated emulsions, and foams. Again our objective is to scrutinize the generic behaviors that emerge from twenty years of continuous research.

\subsection{Microgel suspensions}

Microgels form a very important class of particles, both for fundamental science and applications. They are made of an intramolecular crosslinked polymeric network swollen by a solvent (Wyss et al 2011). The size of individual particles spans several orders of magnitude, from $10 \mathrm{~nm}$ to $1 \mu \mathrm{m}$ or more. Neutral or polyelectrolyte water-soluble microgels offer the richest opportunities in terms of environmentally safe applications. A popular example of neutral water-swellable microgels is poly( $N$-isopropylacrylamide) (PNIPAm) thermosensitive particles (Meyer and Richtering). Polyelectrolyte microgels include colloidal particles made from poly(methylmethacrylic acid) (Eichenbaum et al 2000), and copolymers of methylmethacrylic acid with methylmethacrylate (Saunders et al 1997) or ethylacrylate (Cloitre et al 2003). Physically crosslinked polymers are also used to create biocompatible microgels (Adams et al 2004; Shewan et al 2017). In microgels the softness and deformability of individual particles is ultimately related to their crosslink density and their degree of swelling.

A series of paper provide a systematic and comprehensive study of wall slip phenomena of well characterized submicron poly(methylmethacrylic acid-ethylacrylate) microgels (Meeker et al 2004a, 2004b; Seth et al 2008; Seth et al 2012; Vayssade et al 2014) in rheometric shear flows using cone and plate or parallel plate geometries and pressure driven flows.

For stresses below the yield stress, the slip behavior was shown to depend on the surface chemistry:

(i) Hydrophobic surfaces favor weak attractive particle-surface interactions resulting in a finite slip yield stress $\sigma_{\mathrm{S}}$. The relationship between the slip velocity and the stress is of the 
form of Eq 3 with $m=2$. When $\sigma_{\mathrm{S}}<<\sigma_{\mathrm{W}}<\sigma_{\mathrm{y}}$, it reduces to the quadratic slip law $V_{S}=\beta \sigma_{w}^{2}$ with $\beta=V^{*} / \sigma_{y}^{2}$, where $V^{*}$ is the slip velocity at the bulk yield stress $\sigma_{\mathrm{y}}$. Note that here the value of the slip exponent is not associated with a non-Newtonian viscosity law like in Kalyon (2005). Figure 5 shows that $V^{*}$ and $\sigma_{\mathrm{S}}$ exhibit remarkable variations with the storage modulus of the suspensions, $G_{0}$, the solvent viscosity, $\eta$, and the particle radius, $R$ : $V^{*} \sim G_{0} R / \eta$ and $\sigma_{S} \sim G_{0}^{0.68}$. In contrast with the case of hard sphere suspensions in Fig. 4 , the entropy $k T / R^{3}$ does not play any role here. For the sake of comparison with the slip behavior of foams and emulsions in the next section, it is interesting to express the slip velocity in terms of the non-dimensional elastic number $\Lambda=\eta V_{S} / G_{0} R$ which characterizes the relative importance of viscous and elastic forces:

$$
\sigma_{W} \approx \sigma_{S}+\sigma_{y} \Lambda^{\frac{1}{2}}
$$

(ii) Hydrophilic surfaces result in repulsive interactions, the solid boundaries being preferentially wetted by a film of water. The slip yield stress is very small and the slip law is linear: $V s=\beta \sigma_{W}$. The wall shear stress has the form:

$$
\sigma_{W} \approx \sigma_{y} \Lambda
$$

For stresses above the yield stress, less data is available. A linear slip law with an exponent $m=1$ independently of the nature of the surfaces has been reported (Seth et al 2012; Vayssade et al 2014). In order to clarify the slip behavior of soft particle suspensions in the yielding regime, Divoux et al (2015) have investigated thermosensitive PNIPAm microgel suspensions. By changing the temperature, they explored a wide range of volume fractions across the jamming transition and found that the slip exponent increases continuously $m=1$ for dilute suspensions to $m=2$ for jammed suspensions. In these experiments changes in particle-wall interactions with temperature were not taken into account, which may affect the interpretation.

\subsection{Gel particle suspensions}

Carbopol gel particles have been extensively used as a model system to investigate the slip properties of soft particles (Piau 2007; Davies and Stokes 2008; Pérez-González et al 2012; Geraud et al 2013; Poumaere et al 2014; Aktas et al 2014; Jofore et al 2015; Ahonguio et al 2016; Ortega-Avila et al 2016). Although Carbopol particles have a relatively ill-defined structure, they are generally considered as crosslinked poly(acrylic acid) gel particles with a size of a few microns (Lidon et al 2016). Whereas surface roughness is generally known to 
inhibit wall slip, it has been reported that Carbopol suspensions can slip onto rough surfaces when the size of the corrugations is smaller than the particle size (Geraud et al 2013). The slip properties of Carbopol suspensions conform to the general picture discussed in the previous paragraph for polyelectrolyte microgels and summarized in Eqs 7 and 8. Below the yield stress, a linear slip law $(m=1)$ is obtained for glass (hydrophilic) surfaces and a quadratic law $(m=2)$ for polymeric (hydrophobic) surfaces (Pérez-González et al 2012; Aktas et al 2014; Ahonguio et al 2016; Ortega-Avila et al 2016). Above the yield stress, the slip exponent is generally equal to $m=1$ (Pérez-González et al 2012; Poumaere et al 2014; Aktas et al 2014) although values of about 2 have been reported (Jofore et al 2015; Ortega-Avila et al 2016).

\subsection{Microscopic modeling}

Concentrated dispersions of microgel and gel particles are modeled as elastic spheres of radius $R$ and Young modulus $E$ closely packed into a disordered jammed configuration (Seth et al 2012). The volume fraction exceeds close-packing so that the spheres are compressed and deformed against their neighbors. The general microstructural theory for slip in these systems is based on the behavior of particles near the wall (Meeker et al 2004a, b). In the absence of flow, the soft particles are pressed against the wall in Hertzian contacts by the osmotic pressure of the bulk suspension. Due to the proximity of the particle and the wall, the particles are sensitive to various short-range forces such as dispersive forces, steric hindrance, electrostatic contributions, and hydrophobic-hydrophilic forces (Seth et al 2008). Below the yield stress, there are no large-scale rearrangements, and in first approximation the particles are locked in their position and do not rotate. When a particle slides along the wall during flow, a thin layer of the solvent exists between the particle and the wall allowing the concentrated dispersions to slip. The thickness of this solvent layer and the resulting shear stress at the wall is set generally by a balance among the forces of hydrodynamic flow, elastic deformation of the particle and attraction and repulsion between the particle and the wall (Meeker et al 2004a, b; Seth et al 2008).

Figure 6 shows a schematic of the lubricated zone where a solvent layer is sandwiched between the microgel particle and the wall. Due to proximity of the interfaces, significant short-range forces between the microgel and the wall may exist. The governing equations for elastohydrodynamics in the thin film coupled with the particle-wall forces are (Seth et al 2008): 


$$
\begin{gathered}
\nabla \cdot\left(\delta^{3} \nabla p\right)=-6 \eta V_{S} \frac{\partial \delta}{\partial x}, \\
\delta(x, y)=-h_{0}+\frac{x^{2}+y^{2}}{2 R}+w(x, y), \\
w(x, y)=\frac{1}{G_{\mathrm{P}}} \int_{-\infty}^{+\infty} \int_{-\infty}^{+\infty} \frac{p+p_{d}(\delta)}{\sqrt{(x-\xi)^{2}+(y-\theta)^{2}}} d \xi d \theta,
\end{gathered}
$$

where $\eta$ is the solvent viscosity. Both $(x, y)$ and $(\xi, \theta)$ are Cartesian coordinates in the plane of the wall; the origin is located on the wall beneath the centre of the particle. $\nabla$ is the two dimensional gradient operator in the plane of the surface. $\delta(x, y)$ is the gap height, $p(x, y)$ is the hydrodynamic pressure in the gap and $p_{d}$ is the disjoining pressure in the gap due to the attractive and repulsive forces. $G_{P}=\pi E /\left(1-v^{2}\right)$ is the contact elastic modulus of the particle ( $E$ is the Young's modulus and $v$ the Poisson's ratio of the particle). Equation (10) describes the flow of solvent through the lubricated gap of thickness $\delta$. Equation (11) defines the particle geometry or gap height $\delta$ as the sum of the undeformed sphere shape and the elastic deformation $w(x, y)$. Equation (12) computes the linear elastic deformation as a function of the net force due the hydrodynamic pressure field $p$ beneath the particle and the disjoining pressure $p_{\mathrm{d}}$ associated with the short range forces. This set of equations can be solved numerically or by scaling analysis. Two regimes of slip are predicted depending on the nature of the particle wall interactions.

\section{Elastohydrodynamic slip}

At rest, the particles stick to the wall when the particle-wall interactions are attractive. Above the slip yield stress, a lubricating slip layer forms according to the mechanism of elastohydrodynamic lubrication. The particle deformation is coupled to the flow through the pressure field so that the flat contacts existing between the particles and the surface are deformed asymmetrically. This breaks the reversibility of the Stokes equation and generates a lift force pushing the particles away from the moving surfaces. The balance between the lift force and the bulk osmotic forces sets the thickness of the lubricated layer. The film thickness and slip velocity determine the drag force on the particle, which is proportional to the stress at the wall.

Figure 7a shows typical dimensionless film thickness and pressure along the center of the particle in the flow direction, which are computed numerically. The particle flattens due to the 
high fluid pressure in the film and the disjoining pressure. Underneath the approximate center of the particle, the pressure is positive. At the rear of the particle, the pressure takes negative values. This region of negative pressure distorts the particle aft and creates the pressure gradient that maintains flow in the lubricating film. Figure $7 \mathrm{~b}$ shows typical predictions of the slip velocity versus the wall stress, which are in good agreement with experimental data. A scaling analysis leads to the slip law found experimentally: $V_{S}=\beta \sigma_{w}^{2}$. It is easy to show that the quadratic form is associated with a nonlinear increase of the lubricated film thickness with the slip velocity: $\delta=R \Lambda^{1 / 2}\left(G_{0} / G_{P}\right)^{1 / 2}$. The value of the yield stress $\sigma_{\mathrm{S}}$ is controlled by the nature of the attractive forces; for van der Waals forces, $\sigma_{S} \sim G_{0}^{3 / 4} A^{1 / 4} / R^{3 / 4}$, where $A$ is the effective Hamaker constant between the microgel and surface with a water layer in between. This prediction is also in good agreement with the experimental result $\sigma_{S} \sim G_{0}^{0.68}$ for microgel suspensions (Fig b). In practice van der Waals interactions, which promote particle-wall adhesion and thus reduce slip, can be varied by tuning the refractive index mismatch between the particles and the solvent (Seth et al 2008).

\section{Hydrodynamic slip}

When the net particle-wall interactions are purely repulsive, the solid boundaries are preferentially wetted by a film of solvent even in the absence of any motion. Slip is possible for very slow flows, indicating that the slip yield stress is very small. The lubricating film thickness for weak flows corresponds to a balance between the bulk osmotic forces and the short-range repulsive forces, and is constant independently of any elastohydrodynamic contribution. This is clearly seen in Fig 8a where the particle facet is nearly flat and symmetric. Since the film has a constant thickness the slip law is linear as demonstrated in Fig. 8 b. Note that at large stress, elastohydrodynamic contributions can take over depending on the range of the repulsive forces.

\section{Slip phenomena in emulsions and foams}

Emulsions and foams are dispersions of one liquid or gas phase stabilized by molecular or polymeric surfactants into a continuous liquid phase. They can be considered as soft particle dispersions but the presence of interfaces brings an additional degree of complexity.

\subsection{Concentrated emulsions}

At high concentration, they acquire solid-like properties and are prone to slip when sheared along solid surfaces like other yield stress materials. There is a rich literature reporting on wall slip in many different emulsion systems used in applications (Ma and Barbosa-Cánovas 
1995; Franco et al 1998; Gallegos and Franco 1999; Plucinski et al 1998; Bertola et al 2003, Pal 1998, 2000) or in fundamental studies (Princen 1985; Salmon et al 2003b; Bécu et al 2004; Seth et al 2012; Mansard et al 2014; Paredes et al 2015). However much less quantitative information about the slip properties of emulsion systems than for microgel and gel particles suspensions are available.

Mansard et al (2014) have observed that relatively monodisperse emulsions can slip onto rough surface patterns due to the built up of a specific stratification at surfaces. Salmon et al (2003b) and Bécu et al (2004) have performed a systematic study of the slip properties of model emulsions sheared along smooth surfaces. Both dilute and jammed emulsions in the yielding regime were shown to slip but different slip laws are reported. For dilute emulsions, the relation between the slip velocity and the wall stress is linear $(m=1)$. The slip layer being much larger than the droplet diameters, slip was associated to migration effects. For jammed emulsions above the yield stress a quadratic slip law is found $(m=2)$.

Seth et al established a link between the slip properties of concentrated and microgel suspensions (Seth et al 2012). They showed that the form of the slip law, i.e. linear or quadratic and the existence of a slip yield stress is also dependent on the nature of the dropletwall interactions as for microgel and gel particle suspensions (Section 3). In many cases the predictions for the slip behavior of emulsions can be mapped onto that of microgel suspensions by changing the contact modulus of the microgel particle by the equivalent value for a liquid drop based on its surface tension.

\subsection{Foams}

Foams are dense dispersions of gas bubbles stabilized by surfactants in a liquid forming a continuous phase. They are prevalent in the food and personal care industry, many manufacturing processes, oil recovery, froth flotation, nuclear decontamination. The existence and control of foam slip at surfaces and interfaces is central to applications. Thus they exhibit many similarities with concentrated emulsions discussed above. One advantage of foams is to be formed from bubbles of macroscopic size that can be easily observed and manipulated. This probably explain why slip phenomena in foams have been examined in detail at different scales, starting from individual bubbles (Bretherton 1961; Kraynik 1998; Emile et al 2009, 2012; Cantat 2013; Germain and Le Merer 2016) to 2D monolayers and 3D assemblies (Cantat et al 2004; Denkov et al 2005, 2006; Terriac et al 2006; Marze et al 2008; Ireland and Jameson 2009; Cohen-Addad and Höhler 2014; Le Merrer et al 2015) 
The slip properties of foams depend on a number of parameters: the liquid viscosity $\eta$, the liquid-gas interfacial tension $\gamma$, the bubble size, the volume fraction $\phi$, and the surfactant type which determines the surface elasticity and viscosity mobility of bubbles. The slip velocity $V_{\mathrm{S}}$ is generally expressed in non-dimensional form using the capillary number $C a=\eta V_{\mathrm{S}} / \gamma$.

One important result that distinguishes foams from other soft materials is that the wall stress between a dry foam $(\phi>0.9)$ and a smooth wall depends on the mobility of the interface (Denkov et al 2005, 2006; Marze et al 2008). The mobility depends on the chemical properties and concentration of surfactants (Golemanov et al 2008; Denkov et al 2009; Emile et al 2009). Surfactants with low and moderate surface modulus are responsible for mobile interfaces whereas surfactants or mixtures with high surface modulus result in immobile interfaces. Different slip laws have been reported depending on the surfactant mobility (Marze et al 2008; Denkov et al 2005, 2006, 2009):
Mobile interfaces
$\sigma_{W} \approx \frac{\gamma}{R} C a^{\frac{2}{3}}$
$V_{S}=\beta \sigma_{w}^{\frac{3}{2}}$
Rigid interfaces
$\sigma_{W} \approx \frac{\gamma}{R} C a^{\frac{1}{2}}$
$V_{S}=\beta \sigma_{w}^{2}$

Since the scale of the elastic modulus of foams is the Laplace pressure $\gamma / R$, the elastic number $\Lambda$ and the yield stress $\sigma_{\mathrm{y}}$ are proportional to the capillary number $C a$ and $\gamma / R$, respectively. Hence expressions (13) and (14) can be mapped onto those for microgel and gel suspensions (Eqs 8 and 9), emphasizing the strong similarities shared by the slip properties of soft materials. For wet foams, the bubbles remain almost spherical and the stress law is linear: $\sigma_{W} \approx C a$ or $V_{\mathrm{S}} \approx \beta \sigma_{W}$. The thickness of the liquid films has been measured and found to be also power law functions of the capillary number (Tisné et al 2004; Emile et al 2012). When the solid surface is not perfectly wetted by the interstitial liquid, the foam-wall interface may exhibit a slip yield stress (Ireland and Jameson 2009).

\subsection{Microscopic modeling}

In a classical paper Bretherton (1961) studied the friction exerted by a long bubble with mobile interface along a solid wall. He assumed that in the central zone of the wetting film the liquid moves like a plug flow, i.e. without no viscous dissipation, and that the friction comes only from the front and rear edges of the film, leading to a slip law of the form: $\sigma_{W} \approx C a^{2 / 3}$. Later on this result has been generalized to dry 3D foams slipping along solid walls (Cantat $e t$ al 2004). 
Denkov et al $(2005,2006)$ considered the case of rigid interfaces which can sustain tangential stress, leading to shear flow in the wetting films in contact with the wall and an additional contribution to the wall stress. The analytical treatment is similar to that for elastohydrodynamic slip in soft particle suspensions also leading to $\sigma_{W} \approx C a^{1 / 2}$. The total wall stress is then the sum of a term originating from the dissipation in the film edges the Plateau borders), $\sigma_{W} \approx C a^{\delta}$, and the term due to the friction in the wetting films, $\sigma_{W} \approx C a^{1 / 2}$. Recently the question of the dissipation in the wetting film was revisited using Bretherton's approach and a new scaling was proposed: $\sigma_{W} \approx C a^{1 / 3}$ (Cantat et al 2013). Unfortunately, the experimental data are equally described by both sets of expressions so that it is impossible to draw a definite conclusion. For wet foams, the dissipation between the bubble and the wall is due to Stokes flow, like in hard sphere suspensions, and a generalized slip law including this contribution, $\sigma_{W} \approx C a$ and the dissipation in the edges, $\sigma_{W} \approx C a^{2 / 3}$ has been proposed (Le Merrer et al 2015).

In conclusion, foams appear to be very specific in the sense that it is necessary to account for the dissipation in the wetting foams and the Plateau borders. This peculiarity does not seem to be shared by emulsions in view of the data available. More systematic investigations on emulsions prepared using surfactants providing different surface mobilities would be interesting to go further.

\section{Curing and controlling wall slip}

Experimentalists have been interested in developing practical solutions for suppressing and/or controlling wall slip in applications. The motivation is to eliminate artifacts during the rheological characterization of yield stress materials since important wall slip can lead to an erroneous determination of the yield stress (see Fig 2a). In other instances, it is sometimes useful to promote wall slip by designing surfaces with specific properties.

\subsection{Using specific geometries to avoid slip artifacts in rheometry}

The vane geometry also called vane-in-cup geometry has been developed and is widely used to measure the rheology of yield stress materials and other structured fluids (Barnes and Nguyen 1991). The vane geometry, originally developed by Dzuy and Boger, is now commercially available from most manufacturers of rheometers (Dzuy and Boger 1983; Dzuy and Boger 1985). In this method, a vane comprising several vertical blades is immersed in a cylindrical cup containing the material to be studied. In the original version, the vane was rotated slowly at a constant rate and yielding was detected when the torque exerted on the 
vane shaft reached a maximum value. Due to the configuration of the geometry, the material in between the vane blades is assumed to move with the vane as a solid and yielding occurs at the perimeter of the cylindrical volume defined by the blades, which considerably reduces or even suppresses the occurrence of slip. The flow field in the vane geometry is relatively complex and ill defined. The method thus relies on several assumptions such as the absence of secondary flows between the blades (Keentok et al 1985). The vane geometry has been used for rheometry of various complex fluids (Meeten and Sherwood 1992; Roberts and Barnes 2001; Baravian et al 2002; Stickland et al 2015). For more details about applications, we refer the interested reader to Barnes and Nguyen (2001). Recent developments have proposed the extension of Small and Large Amplitude Oscillatory Shear rheology to the vane geometry (Patarin et al 2014). Other alternative geometries like helicoidal tools (Cullen et al 2003) or serrated plates (Zhu et al 2001) have been invented to characterize yield stress materials.

\subsection{Implementing physically textured surfaces}

Rough shearing surfaces are commonly used to suppress wall-slip (Magnin and Piau 1990). The current interpretation is that the asperities of the surfaces disrupt the lubricating film responsible for slip. This can be achieved by sandblasting (Buscall et al 1993; Mason et al 1996; Meeker et al 2004a, b; Gibaud et al 2008; Lettinga and Manneville 2009) or machining (Magnin and Piau 1990; Gulmus and Yilmazer 2005) the surfaces of the geometries, by sticking solvent proof sandpaper (Khan et al. 1988; Coussot et al 2002; Piau 2007; Divoux et al 2012; Ahonguio et al 2016) or by creating specific grooved of serrated tools (Nickerson and Kornfield 2005 ; Mansard et al 2014). Kalyon and his co-workers used aluminium oxide and silicon carbide impregnated shearing surfaces to prevent the occurrence of wall slip in concentrated suspensions (Kalyon et al 1993; Aral and Kalyon 1994). Slip can also be eliminated by gluing or sintering a concentrated, disordered layer of particles deposited onto the surfaces (Kao et al 1975; Marze et al 2008; Besseling et al 2009).

Even though these techniques are useful and operate in most cases, they remain empirical and in most cases it is not possible to determine the appropriate texture or level of surface roughness needed to remove slip. Khan et al (1988) used sandpaper with variable grit sizes stuck on the shearing surfaces to suppress wall slip in foams. They found that slip reduction was optimum when the sandpaper grit size was commensurate with the bubble size. Gulmus and Yilmazer (2007) proposed to characterize the roughness by the arithmetic average height of the texture profile, $R_{\mathrm{a}}$. Aral and Kalyon (1994) performed a systematic evaluation of the effect of roughness on the development of wall slip in non-aggregating 
concentrated suspensions. They studied particle size to $R_{\mathrm{a}}$ ratios, $R / R_{\mathrm{a}}$, ranging from 230 to 0.3. Slip was found to decrease with decreasing $R / R_{\mathrm{a}}$, slip being suppressed when the particle size was of the order of the roughness parameter, i.e. $R / R_{\mathrm{a}} \cong 1$. The influence of varying degree of roughness was also examined in detail in relation with the squeeze rheometry of soft dispersions (Meeten GH 2004a, b). Slip was lessened or removed by plate roughness but perfect slip was not approached by any material, even when squeezed by optically polished plates. Recently Mansard et al (2014) designed well-controlled surface patterns and studied the impact of roughness on microfluidic flows of concentrated emulsions. They used a standard photolithography technique to imprint parallelepiped pillars of variable heights regularly arranged on glass surfaces. They found that the slip velocity exhibited a nonmonotonic variation: it first decreased until the roughness becomes comparable to the radius of the emulsion droplets, in agreement with the previous observations reported above, and increased beyond that point. They attributed the increase of the slip velocity to the formation of an ordered second layer of droplets above the corrugations.

The studies reported above converge towards the conclusion that the optimum roughness value is the particle size when the particles are well dispersed. This result is reminiscent of similar observations for polymer solutions (Sanchez-Reyes and Archer 2003). In these systems the effective slip interface in these systems is located a distance of the order of the radius of gyration $R_{\mathrm{g}}$ of the polymer coils from the surface and slip is eliminated when the root-mean-squared roughness of the surfaces is smaller than $0.65 R_{\mathrm{g}}$. However, the situation is far more complex when attractive interparticle interactions lead to the formation of colloidal gels. A recent study considered the case of colloidal gels formed under polymer induced depletion attraction between Brownian PMMA particles (Ballesta et al 2013). These attractive gels slip even for surfaces of roughness similar to the individual particle size. This is caused by the fractal nature of the gels which is responsible for a reduction of the number of bonds between bulk and surface as the cluster size increases. To eliminate slip, the roughness has to be much larger than the particle size, comparable to the size of the largest heterogeneities, here the aggregates, which are present in the system.

\subsection{Tuning surface chemistry}

Depending on their chemical nature, shearing surfaces can be made either attractive or repulsive with respect to the particles of the dispersion (Seth et al 2008). Surfaces which are totally wetted by the continuous phase are responsible for the formation of a continuous lubricating film: the particles of the dispersed phase are depleted from the wall which is in 
favor of wall slip. On the opposite, particles tend to stick on non-wetting surfaces, which contribute to create some artificial roughness and limit slip. In their study of colloidal gels, Walls et al observed that hydrophobic silica particles are repelled by hydrophilic surfaces in favor of the polar solvent whereas they are attracted by hydrophobic plates, thus reducing slip (Walls et al 2003). Similarly, it was observed that hydrophilic plates have minimal effect on the slip of oil in water emulsions but that the oil droplets stick to and even coalesce at hydrophobic surfaces (Mannheimer 1972; Princen 1985). When the refractive index of the particles and the suspending medium are matched, van der Waals forces are reduced and insufficient to make the particles adhere to the surface, which is in favor of slip (Ballesta et al 2012; Seth et al 2012).

Hence slip can be manipulated by chemically modifying or patterning the shearing surfaces. The control of wall slip by changing the surface chemistry remains scarcely explored, but there are some indications that it may be a promising route. In some cases, adhesion between shearing surfaces and the materials has been promoted using specific adhesives (Magnin and Piau 2007). Seth et al (2008, 2012) grafted a cationic silane coating on glass plates to suppress the wall slip of anionic microgel pastes. On the same line Métivier et al (2012) used an adsorbed layer of cationic polyethyleneimine on PMMA to inhibit wall slip of polacrylic Carbopol. The recent invention of liquid impregnated surfaces that have unique repellent properties could provide alternate solutions and prompt further developments (Solomon et al 2014).

\section{Open questions for future research}

In this review, we have revisited important topics related to wall slip in high solid dispersions: detection, generic signatures in rheometry and pressure driven flows, slip phenomena in hard and soft particle suspensions, advances in microscopic modeling, and methods to avoid or correct wall slip. In this concluding section we pose some open questions that emerge from recent experimental and theoretical investigations of yield stress materials.

\section{Wall slip as a mechanism to relax elastic stresses}

Section 5 describes different techniques to eliminate wall slip, in particular the use of rough surfaces or chemically modified surfaces promoting adhesion. In some instances, bulk flow heterogeneities such as shear banding or fracture take over wall slip when the latter is effectively suppressed leading to interesting phenomena not yet fully understood. One of the earlier observations of the competition between wall slip and shear banding was made by 
Persello et al (1994). Concentrated suspensions of colloidal silica particles were shown to exhibit wall slip. When slip was suppressed, direct visualization revealed that the deformation was localized in one or several slip layer in the bulk of the suspensions; the slip layers were healing upon flow cessation. Similar observations have been reported in nanofibrillated cellulose suspensions in simple shear flows (Nechyporchuk et al 2014). Whereas wall-slip was detected at low shear rates with smooth cone and plate geometry, the roughening of the tool surfaces was accompanied by the appearance of shear banding and the use of serrated tools provoked water release from the suspension. Hard sphere glasses in the absence of slip show a particular type of shear banding caused by instability due to shear concentration coupling (Besseling et al 2010). In non-Brownian suspensions, Aral and Kalyon (1994) also noted that increased surface roughness prevents slip but results in fracture.

Similar observations have been reported in polymeric and surfactant solutions indicating that the phenomenon may be general. For instance, wormlike micelles exhibit standard shear banding signaled by a stress plateau in the flow curve for adhesive boundary conditions, but undergo wall slip at shear rates larger than the start of the stress plateau for non-adhesive surfaces (Lettinga and Manneville 2009). In some systems, wall slip and shear banding can occur in competition depending on the material composition, flow rate, and detailed experimental conditions (Feindel and Callaghan 2010; Boukany and Wang 2010; Manneville et al 2007; Jaradat et al 2012; Martoïa et al 2015). These observations question the relation between wall slip and shear banding flows. The development of large scale flow heterogeneities seems to be associated to the existence of a limiting internal stress that the materials cannot accommodate without relaxing excess elastic energy either locally via wall slip or at large scale through shear bands formation. The boundary conditions then may play an important role in favoring one particular scenario (Adams et al 2008). Clearly more investigations are needed to get a full understanding of the problem and build a predicting framework.

\section{Non locality mediated by surface roughness}

Recent studies have shown that the flow behavior of concentrated yield stress materials is characterized by non-locality, i.e. the relation between the stress and the shear rate is not given by a unique constitutive equation (Goyon et al 2008; Goyon et al 2010; Mansard and Colin 2013). Non-locality is associated with the existence of plastic rearrangements that release elastic waves propagating over a characteristic length scale, termed cooperativity length, of the order of a few particle sizes (Bocquet et al 2009). A consequence is that the 
flow properties become dependent on confinement and surface topography. Hence it is likely that the degree of roughness or smoothness not only locally alters the structure of the material but also controls the plastic rearrangements that create a mechanical noise propagating inside the bulk material. Several experimental studies have reported results in the direction that rough surfaces affect surface fluidization in confined flows (Goyon et al 2008; Goyon et al 2010; Mansard et al 2014; Geraud et al 2013). A recent study analyzes the importance of surface texture with respect to surface fluidization by changing the amplitude and wavelength of the corrugations created by specific surface patterns (Derzsi et al 2016). Still more surprising significant alterations of the velocity profiles over distances to the wall much larger than a few particle sizes have been reported when surfaces are smooth and develop attractive interactions with the particles of the dispersions (Seth et al 2012; Paredes et al 2015). These observations, once correctly understood and modeled, could open new routes to control bulk flows in microfluidic devices.

\section{Slip driven flows}

As an extreme possible manifestation of non-locality, it has been found that the existence of a slip layer can control the entire flow in some complex yield stress materials. This is the case of micro-fibrillated cellulose suspensions which exhibit a multilayer flow structure comprising a few micron thick slip layer near the walls, a wall boundary layer where the velocity is larger than in the bulk, and a plug region where the material does not deform (Haavisto et al 2015). The formation of this specific flow structure could be explained in terms of fluidization induced by a strong shearing motion induced by wall slip (Nazari et al 2016). We note that here strong slip is considered to be at the origin of fluidization rather than plastic noise induced by surface corrugations and asperities like in the previous section.

That wall slip can influence the fluidization of a yield stress material initially at rest has also been discussed in Laponite suspensions sheared under different boundary conditions (Gibaud et al 2008). Rough walls ensuring no-slip boundary conditions are responsible for transient long-lasting shear localization as commonly observed in various high solid dispersions (Divoux et al 2010). With smooth geometries where apparent wall slip is possible, the material loses cohesion by breaking into macroscopic pieces that are progressively eroded by the bulk fluidized material. Full slip also allows for faster stress relaxation upon flow inception in gel particles suspensions (Divoux et al 2011a, b) 
To conclude, wall slip behavior actually constitutes a fundamental component of the flow and deformation behavior of yield stress materials far from being a simple artifact as often considered. The question of how promoting or suppressing wall slip can alter the entire flow of yield stress materials is one of those that deserve much attention. 

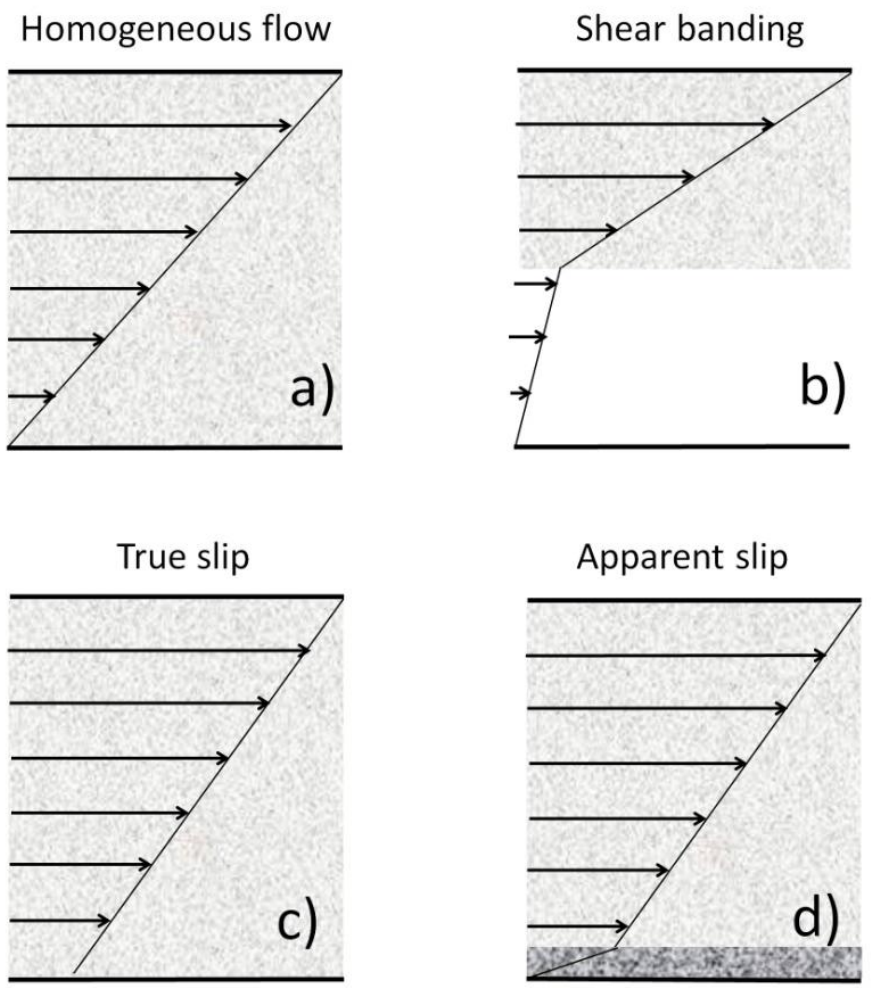

Figure 1: Macroscopic flow structure of high solid dispersions with yielding properties 

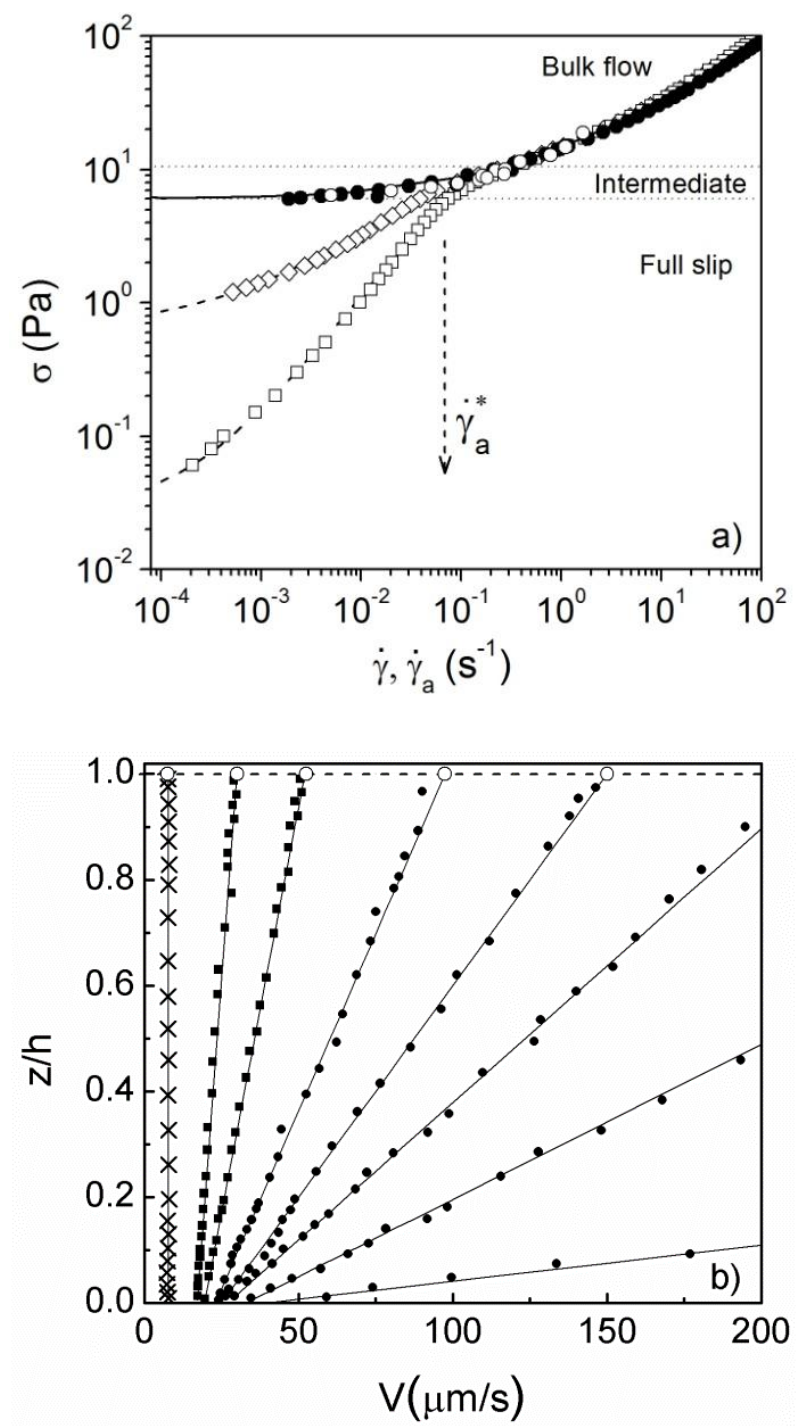

Figure 2: Generic signatures of wall slip represented for a concentrated microgel suspension in parallel plate geometry. a) Variations of the shear stress versus the true shear rate (no slip) or apparent shear rate (slip). When sheared with rough surfaces $(0)$, the suspension exhibits a true yield stress and the flow curve is well described by the Herschel- Bulkley equation (---). With smooth polymer $(\diamond)$ and glass surfaces $(\square)$, which are hydrophobic or hydrophilic respectively, the flow curves exhibits three regimes of flow. $\dot{\gamma}_{a}^{*}$ marks the onset of full slip; the equations of the dashed lines are of the form $\sigma=\sigma_{S}+k \dot{\gamma}_{a}^{m}$ with $\square_{\mathrm{S}}=0.03$ and 0.6 , $m=0.92$ and 0.50 for the glass and polymer surfaces respectively. The local rheology data corrected from the effect of slip coincide with the flow curve in the absence of slip $(\bigcirc) . b$ ) Velocity profiles measured using PTV for the same microgel suspension as in a). The top surface is rough and obviates slip; the bottom surface is a polymer surface: $\mathrm{x}$ : full slip regime; $\mathbf{\square}$ : intermediate regime; $\mathbf{0}$ : full yielding with negligible slip. The slope of the velocity profiles gives access to the local rheology data reported in a). The data are reproduced from Seth et al (2012) with permission of authors. 

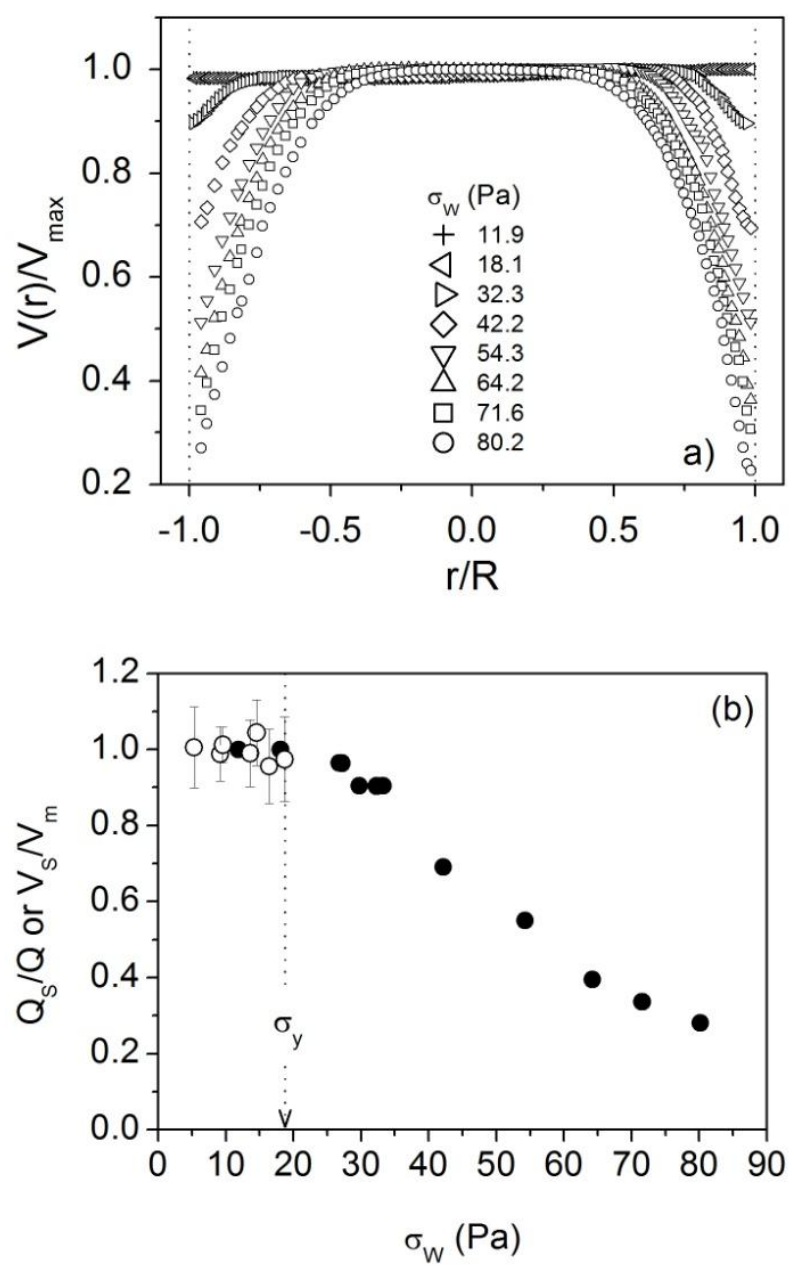

Figure 3: Generic signatures of wall slip in channel flows. The suspension is a dispersion of Carbopol gel particles in distilled water. The capillary has a circular section and is made of borosilicate glass. a) The normalized velocity, $V_{\mathrm{z}}(\mathrm{r}) / V_{\max }$ where $V_{\max }$ is the plug velocity, distributions for increasing values of the wall shear stress $\sigma_{\mathrm{W}}$; $\mathrm{b}$ ) Ratio of volumetric flow rate due to wall slip, $Q \mathrm{~s}$, over flow rate, $Q$, versus shear stress (or $V_{\mathrm{S}}$ versus $V_{\mathrm{m}}$ ) at the edge for steady torsional flow and wall shear stress for capillary flow; $V_{\mathrm{m}}$ is the mean velocity. Data reproduced from Aktas et al (2014) with permission of the authors. 

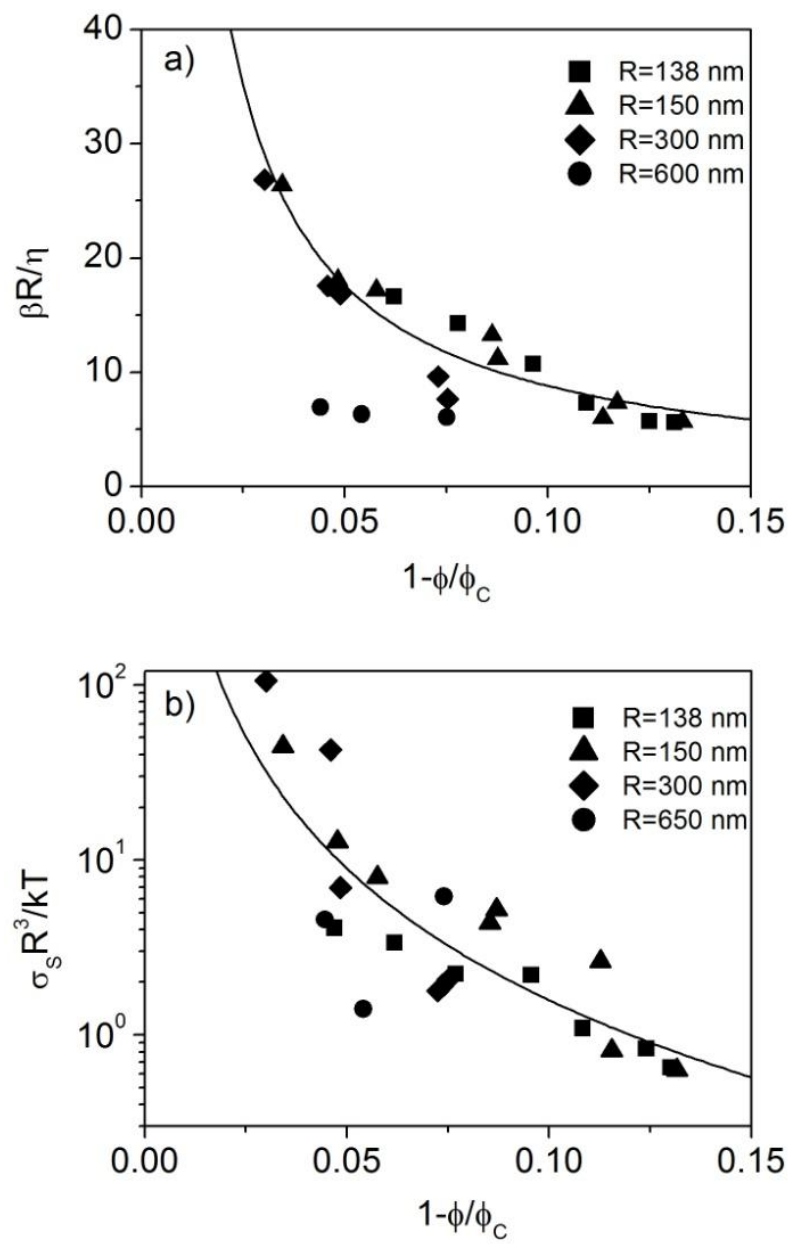

Figure 4: Slip parameters for hard sphere suspensions. a) Normalized lubrication parameter $\beta R / \eta$ versus $1-\phi / \phi_{\mathrm{C}}\left(\phi_{\mathrm{C}}=0.64\right)$. The continuous line represents the empirical expression: $\beta R / \eta \cong 0.9\left(1-\phi / \phi_{m}\right)^{-1}$. b) Normalized slip stress $\sigma_{\mathrm{S}} R^{3} / k T$ versus $1-\phi / \phi_{\mathrm{C}}$. The continuous line represents the empirical equation $\sigma_{S} R^{3} / k T \cong 0.005\left(1-\phi / \phi_{m}\right)^{-2.5}$. Data reproduced from Ballesta et al (2012) with permission of the authors. 

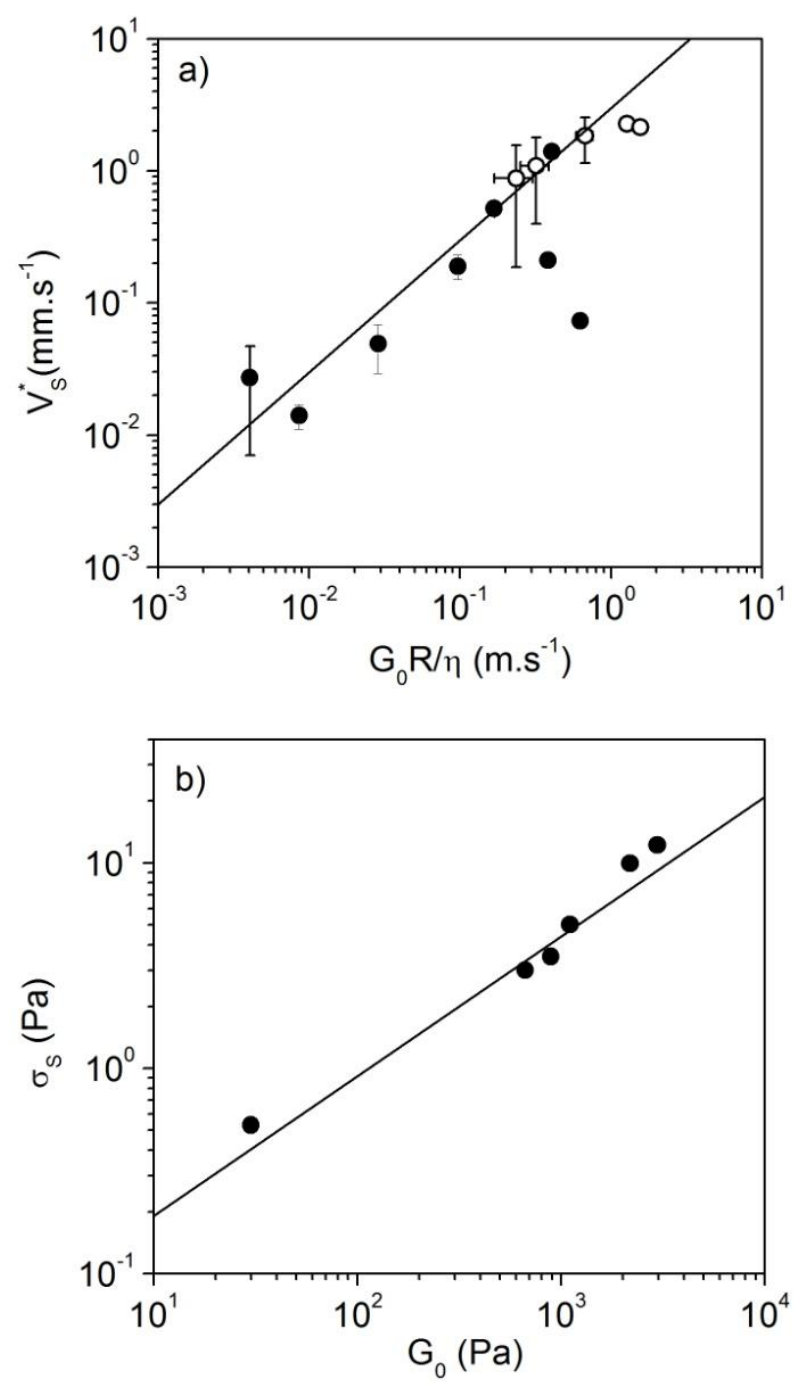

Figure 5: Slip parameters for soft particle suspensions. a) Slip velocity at the yield point, $V^{*}$ for acrylic microgel suspensions $(O)$ and silicon oil in water $(O)$ versus the characteristic velocity $G_{0} R / \eta$. Data reproduced from Meeker et al (2004a,b). b) Scaling of the slip yield stress $\sigma_{\mathrm{S}}$ versus the storage modulus of the suspension for microgel suspensions sheared along gold and silicon wafers. Solid line shows that the slip yield stress increases as $\sigma_{\mathrm{S}} \sim 0.04 G_{0}^{0.68}$. Data reproduced from Seth et al (2012). 

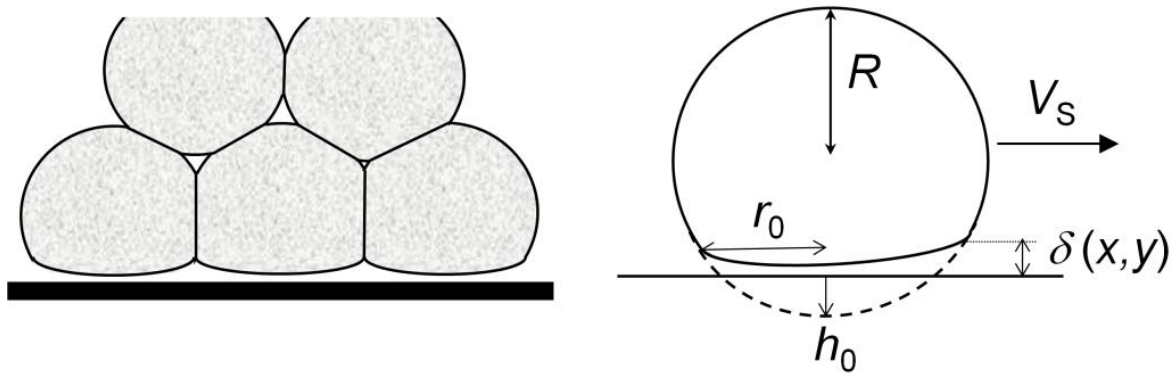

Figure 6: Schematics of a concentrated suspension of soft particles near a smooth surface (left) and detailed view of a particle slipping with velocity $V_{s}$ (right). The compression distance $h_{0}$ and the radius of the contacting facet $r_{0}$ depend on the bulk osmotic pressure and the particle elasticity. From Bonnecaze and Cloitre (2010). 

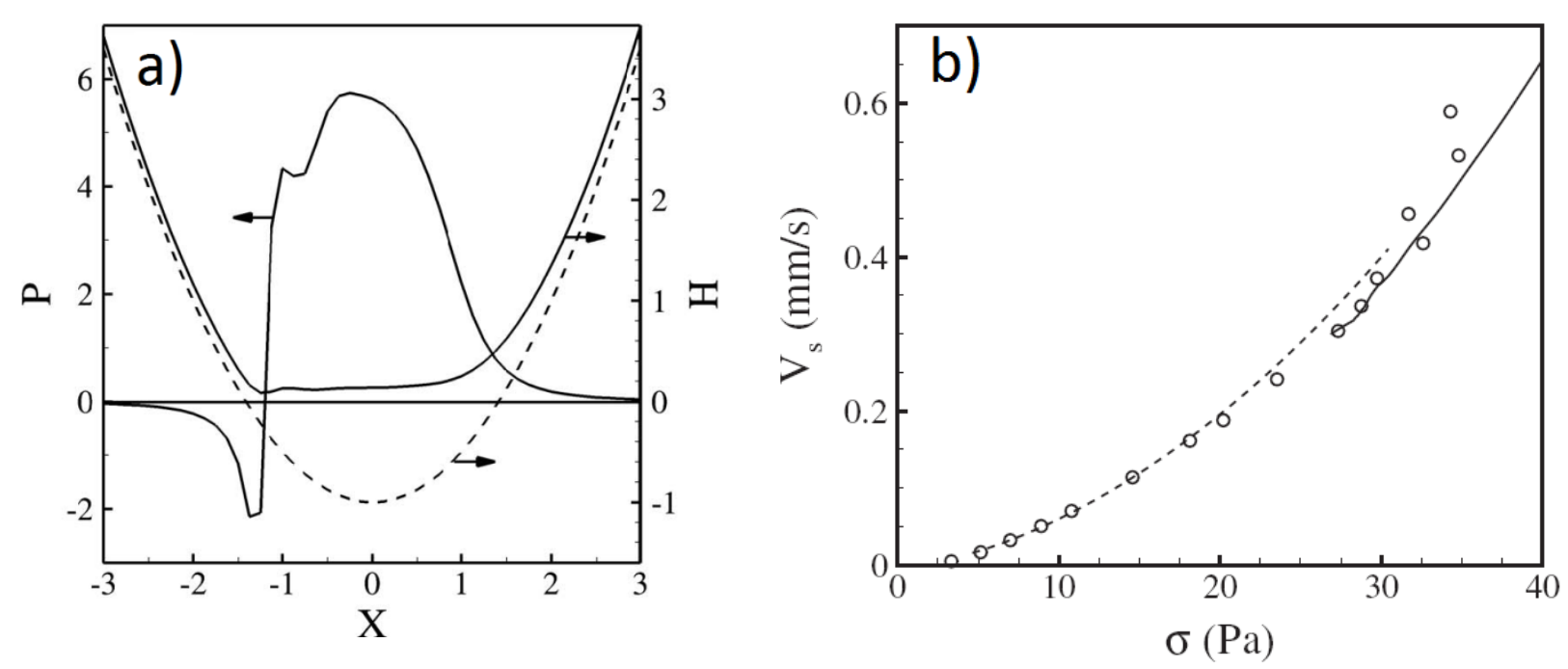

Figure 7: a) Dimensionless pressure $\left(P=p / p_{0}\right)$ and film thickness $\left(\mathrm{H}=\delta / h_{0}\right)$ between particle and wall along the center of the particle in the flow direction $\left(X=x / r_{0}\right)$ are shown for a soft particle slipping along an attractive wall. b) Slip velocity as a function of wall shear stress predicted by the elastohydrodynamic equations with a disjoining pressure (solid and dashed lines) and measured experimentally for a polymeric wall (symbols). Note that the slip velocity increases quadratically with the wall stress and that the slip yield stress is finite. Data reproduced from Seth et al (2008). 

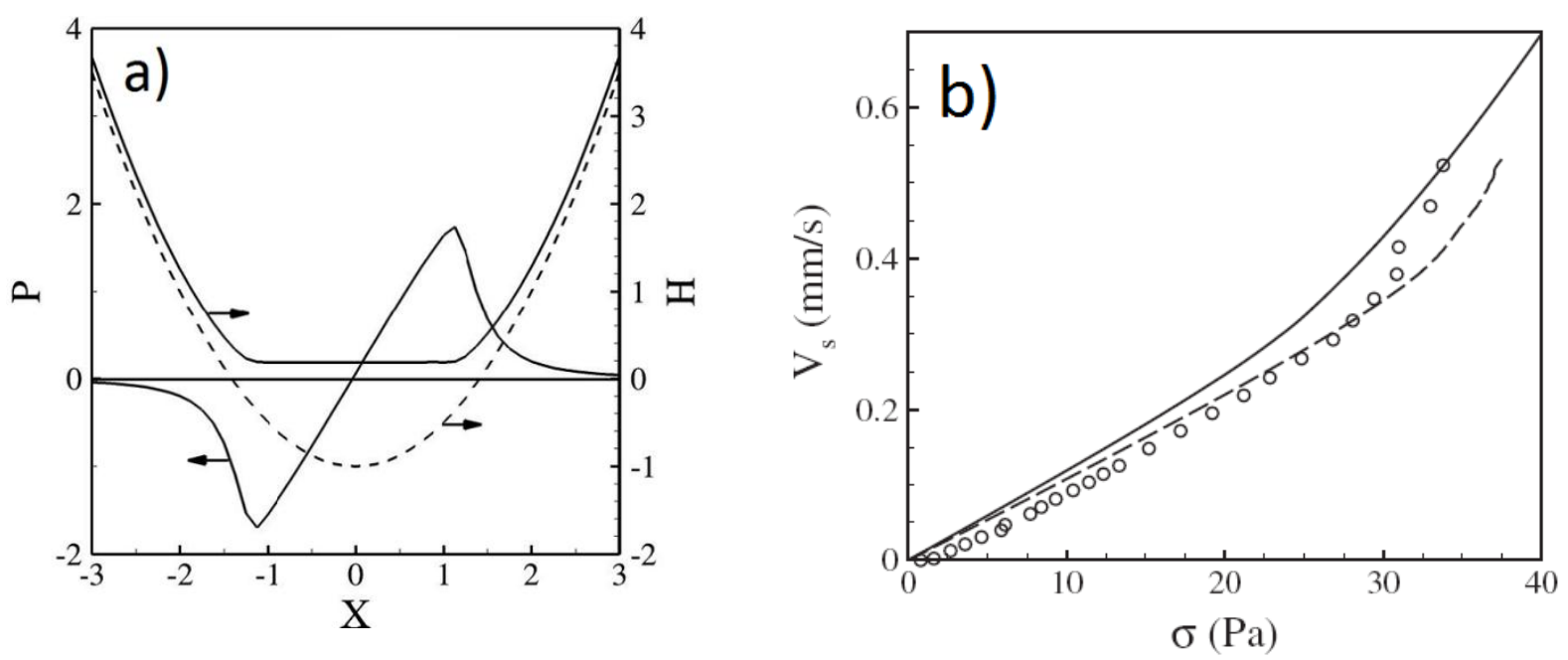

Figure 8: a) Dimensionless pressure $\left(P=p / p_{0}\right)$ and film thickness $\left(\mathrm{H}=\delta / h_{0}\right)$ between particle and wall along the center of the particle in the flow direction $\left(X=x / r_{0}\right)$ are shown for a soft particle sliding along a repulsive wall. Slip velocities as a function of wall shear stress predicted by the elastohydrodynamic equations with a disjoining pressure (solid and dashed lines) and measured experimentally for a silicon wall (symbols). Note that the slip velocity first increases linearly with the wall stress and that the slip yield stress is negligible. Data reproduced from Seth et al (2008). 


\section{References}

Adams S, Frith WJ, Stokes JR (2004) Influence of particle modulus on the rheological properties of agar microgel suspensions. J Rheol 48:1195-1213

Adams JM, Fielding SM, Olmsted PD (2008) The interplay between boundary conditions and flow geometries in shear banding: Hysteresis, band configurations, and surface transitions. J Non-Newtonian Fluid Mech 151: 101118

Ahonguio F, Laurent Jossic L, Magnin A (2016) Influence of slip on the flow of a yield stress fluid around a flat plate. AIChE J 62:1356-1363

Ahuja A, Singh A (2009) Slip velocity of concentrated suspensions in Couette flow. J Rheol 53:1461-1485.

Aktas S, Kalyon DM, Marín-Santibáñez BM, Pérez-González J (2014) Shear viscosity and wall slip behavior of a viscoplastic hydrogel. J Rheol 58:513-535

Allain C, Cloitre M, Wafra M (1995) Aggregation and sedimentation in Cclloidal suspensions. Phys Rev Lett 74:1478-1481

Amin MHG, Hanlon AD, Hall LD, Marriott C, Ablett S, Wang W, Frith WJ (2003) A versatile single-screwextruder system designed for magnetic resonance imaging measurements. Meas Sci Technol 14:1760-1768

Aral BK, Kalyon DM (1994) Effects of temperature and surface roughness on time-dependent developmetn of wall slip in steady torsional flow of concentrated suspensions. J Rheol 38:957-972

Atalıka K, Keunings R (2004). On the occurrence of even harmonics in the shear stress response of viscoelastic fluids in large amplitude oscillatory shear. J Non-Newton Fluid 122:107-116

Baravian C, Lalante A, Parker A (2002) Vane rheometry with a large, finite gap. Appl Rheol 12:81-87

Ballesta P, Petekidis G, Isa L, Poon WCK, Besseling R (2008) Slip and flow of hard-sphere colloidal glasses. Phys Rev Lett 101:258201

Ballesta P, Petekidis G, Isa L, Poon WCK, Besseling R (2012) Wall slip and flow of concentrated hard-sphere colloidal suspensions. J Rheo 56:1005-1037

Ballesta P, Koumakis N, Besseling R, Poon WCK, Petekidis G (2013) Slip of gels in colloid-polymer mixtures under shear. Soft Matter 9, 3237-3245

Barnes HA (1995) A review of the slip (wall depletion) of polymer solutions, emulsions and particle suspensions in viscometers: its cause, character, and cure. J Non-Newtonian Fluid Mech 56:221-251

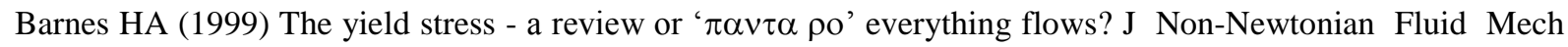
81:133-178

Barnes HA, Nguyen QD (2001) Rotating vane rheometry - a review. J Non Newtonian Fluid Mech 98:1-14

Barnes EC, Wilson DI, Johns ML (2006) Velocity profiling inside a ram extruder using magnetic resonance (MR) techniques. Chem Eng Sci 61:1357-1367

Bécu L, Grondin P, Colin A, Manneville S (2004). How does a concentrated emulsion flow? Yielding, local rheology, and wall slip. Colloids and Surfaces A: Physicochem Eng Aspects 263:146-152

Bécu L, Manneville S, Colin A (2006). Yielding and flow in adhesive and non-adhesive concentrated emulsions. Phys Rev Lett 96:138302

Bertola V, Bertrand F, Tabuteau H, Bonn D, Coussot P (2003) Wall slip and yielding in pasty materials. J Rheol 47:1211-1226

Besseling R, Isa L, Weeks ER, Poon WCK (2009) Quantitative imaging of colloidal flows. Adv Colloid Interface Sci 146: 1-17 
Besseling R, Isa L, Ballesta P, Petekidis G, Cates ME, Poon WCK (2010) Shear banding and flow-concentration coupling in colloidal glasses. Phys Rev Lett $105: 268301$

Bingham EC (1922). Fluidity and plasticity (McGraw-Hill, New York).

Birinci E, Kalyon DM (2006). Development of extrudate distortions in poly(dimethyl siloxane) and its suspensions with rigid particles. J Rheol, 50:313-326

Blondin E, Doubliez L (2002) Particle image velocimetry of a wet aqueous foam with an underlying liquid film. Exp Fluids 32:294-301

Bocquet L, Colin A, Ajdari A (2009) Kinetic theory of plastic flow in soft glassy materials. Phys Rev Lett 103:036001

Bonn D, Denn MM (2009) Yield stress fluids slowly yield to analysis. Science 324:1401-1402

Bonn D, Paredes J, Denn MM, Berthier L, Divoux T, Manneville S (2015) Yield stress materials in soft condensed matter. arXiv:1502.05281v1

Bonnecaze RT, Cloitre M (2010) Micromechanics of Soft Particle Glasses. Adv Polym Sci 236: 117-161

Boukany PE, Wang S-Q (2008) Use of particle-tracking velocimetry and flow birefringence to study nonlinear flow behavior of entangled wormlike micellar solution: From wall slip, bulk disentanglement to chain scission. Macromolecules 41:1455-1464

Boukany PE, Hemminger O, Wang SQ, Lee LJ (2010) Molecular imaging of slip in entangled DNA solution. Phys Rev Lett 105, 027802

Bower C, Gallegos C, Mackley MR (1999) The rheological and microstructural characterisation of the nonlinear flow behaviour of concentrated oil-in-water emulsions. Rheol Acta 38:145-159

Bouzigues CI, Tabeling P, Bocquet L (2008) Nanofluidics in the Debye layer at hydrophilic and hydrophobic surfaces. Phys Rev Lett 101:114503

Bretherton FP (1961) The motion of long bubbles in tubes. J Fluid Mech 10:166-188

Buscall R, McGowan JI, Morton-Jones AJ (1993) The rheology of concentrated dispersions of weakly attracting colloidal particles with and without wall slip. J Rheol 37:621-641

Buscall R (2010) Letter to the Editor : Wall slip in dispersion rheometry. J Rheol 54:1177-1183

Callaghan PT (1999) Rheo-NMR: nuclear magnetic resonance and the rheology of complex fluids. Rep Prog Phys 62:599-670

Callaghan PT (2006) Rheo-NMR and velocity imaging. Curr Opin Colloid Interface Sci 11:13-18

Callaghan PT (2008) Rheo NMR and shear banding. Rheol Acta 47:243-255

Cantat I, Kern N, Delannay R (2004) Dissipation in foam flowing through narrow channels. Europhys Lett 65:726-732

Cantat I (2013) Liquid meniscus friction on a wet plate: Bubbles, lamellae, and foams. Phys Fluids 25:031303

Chen L, Duan Y, Zhao C, Yang L (2009) Rheological behavior and wall slip of concentrated coal water slurry in pipe flows. Chemical Engineering and Processing 48:1241-1248

Chen DTN, Wen Q, Janmey PA, Crocker JC, Yodh AG (2010) Rheology of soft materials. Annu Rev Condens Matter Phys 1:301-322

Cloitre M, Borrega R, Monti F, Leibler L (2003) Structure and flow of polyelectrolyte microgels: From suspensions to glasses. C R Physique 4:221-230

Cloitre M (2011). Yielding, flow and slip in microgel suspensions: Bridging the gap between microstructure and macroscopic rheology, in Microgel Suspensions: Fundamentals and Applications (H. Wyss, A. Fernandez de Las Nieves, J. Mattson, D.A. Weitz Eds; Wiley-VCH) 
Cloitre M, Vlassopoulos D (2011) Block copolymers in external fields: Rheology, flow-induced phenomena and applications. in Applied Polymer Rheology: Polymeric Fluids with Industrial Applications (M Kontopoulou Ed ; John Wiley \& Sons Inc, 2011)

Cohen I, Davidovitch B, Schofield AB, Brenner MP, Weitz DA (2006) Slip, yield, and bands in colloidal crystals under oscillatory shear. Phys Rev Lett PRL 97:215502

Cohen-Addad S, Höhler R (2014) Rheology of foams and highly concentrated emulsions. Curr Opin Colloid Interface Sci 19:536-548

Condre J-M, Ligoure C, Cipelletti L (2007) The role of solid friction in the sedimentation of strongly attractive colloidal gels. J Stat Mech P02010

Coussot P, Nguyen QD, Huynh HT, Bonn D (2002) Avalanche behavior in yield stress fluids. Phys Rev Lett 88: 175501

Coussot P, Tocquer L, Lanos C, Ovarlez G (2009) Macroscopic vs. local rheology of yield stress fluids. J NonNewton Fluid 158:85-90

Coussot P (2014) Yield stress fluid flows: A review of experimental data. J Non-Newtonian Fluid Mech 211:3149

Crocker JC, Grier DG (1996). Methods of digital video microscopy for colloidal studies, J Colloid Interface Sci 179:298-310

Cullen PJ, O’Donnell CP, Houska M (2003) Rotational rheometry using complex geometries-A review. J Texture Stud 34:1-20.

Damianou Y, Philippou M, Kaoullas G, Georgiou GC (2014) Cessation of viscoplastic Poiseuille flow with wall slip. J Non-Newton Fluid 203:24-37

Davies GA, Stokes JR (2008) Thin film and high shear rheology of multiphase complex fluids. J Non-Newtonian Fluid Mech 148: 73-87

Denkov ND, Subramanian V, Gurovich D, Lips A (2005) Wall slip and viscous dissipation in sheared foams: Effect of surface mobility. Colloids and Surfaces A: Physicochem Eng Aspects 263:129-145

Denkov ND, Tcholakova S, Golemanov K, Subramanian V, Lips A (2006). Foam-wall friction: Effect of air volume fraction for tangentially immobile bubble surface. Colloids and Surfaces A: Physicochem Eng Aspects 282/283:329-347

Denkov ND, Tcholakova S, Golemanov K, Ananthpadmanabhan KP, Lips A (2009) The role of surfactant type and bubble surface mobility in foam rheology. Soft Matter 5, 3389-3408.

Derakhshandeh B, Vlassopoulos D, Hatzikiriakos SG (2012) Thixotropy, yielding and ultrasonic Doppler velocimetry in pulp fibre suspensions. Rheol Acta 51:201-214

Derzsi L, Filippi D, Mistura G, Pierno M, Lulli M, Sbragaglia M, Bernaschi M, Garstecki P (2016) Fluidization and wall slip of soft-glassy materials by controlled surface roughness. arXiv:1611.01980v1

Denn MM (2001) Extrusion instabilities and wall slip. Annu Rev Fluid Mech 33:265-87

Divoux T, Tamarii D, Barentin C, Manneville S (2010) Transient shear banding in a simple yield stress fluid. Phys Rev Lett 104: 208301

Divoux T, Barentin C, Manneville S (2011a) From stress-induced fluidization processes to Herschel-Bulkley behaviour in simple yield stress fluids. Soft Matter 7:8409-8418

Divoux T, Barentin C, Manneville S (2011b) Stress overshoot in a simple yield stress fluid: An extensive study combining rheology and velocimetry. Soft Matter 7, 9335

Divoux T, Tamarii D, Barentin C, Teitel S, Manneville S (2012) Yielding dynamics of a Herschel-Bulkley fluid: a critical-like fluidization behaviour. Soft Matter 8:4151-4164 
Divoux T, Lapeyre V, Ravaine V, Manneville S (2015) Wall slip across the jamming transition of soft thermoresponsive particles. Phys Rev E 92:060301.

Divoux T, Fardin MA, Manneville S, Lerouge S (2016) Shear Banding of Complex Fluids. Annu Rev Fluid Mech 48:81-103

Dzuy NQ, Boger DV (1983) Yield stress measurement for concentrated suspensions. J Rheol 27:321-349.

Dzuy NQ, Boger DV (1985) Direct yield stress measurement with the vane method. J Rheol 29:335-347

Egger H, McGrath KM (2006) Estimating depletion layer thickness in colloidal systems: Correlation with oil-inwater emulsion composition. Colloids and Surfaces A: Physicochem Eng Aspects 275:107-113

Eichenbaum GM, Kisetr PF, Simon SA, Needham D (1998) pH and ion-triggered volume response of anionic hydrogel microspheres. Macromolecules 31:5084-5093

Emile J, Salonen A, Dollet B, Saint-Jalmes A (2009) A systematic and quantitative study of the link between foam slipping and interfacial viscoelasticity. Langmuir 25:13412-13418

Emile J, Casanova F, Loas, Emile O (2012) Swelling of a foam lamella in a confined channel. Soft Matter 8:7223-7227.

Ewoldt R, Clasen C, Hosoi AE, McKinley GH (2007). Rheological fingerprinting of gastropod pedal mucus and bioinspired complex fluids for adhesive locomotion. Soft Matter 3:634-643.

Feindel KW, Callaghan PT (2010) Anomalous shear banding: multidimensional dynamics under fluctuating slip conditions. Rheol Acta 49:1003-1013

Fielding SM (2014) Shear banding in soft glassy materials. Rep Prog Phys 77:102601.

Frank M, Anderson D, Weeks ER, Morris J (2003) Particle migration in pressure-driven flow of a Brownian suspension. J Fluid Mech 493:363-378.

Franco JM, Gallegos C, Barnes HA (1998) On slip effects in steady-state flow measurements of oil-in-water food emulsions. J Food Eng 36:89-102

Fujii S, Richtering W (2006) Size and viscoelasticity of spatially confined multilamellar vesicles. Eur Phys J E 19:139-148

Gallegos C, Franco JM (1999). Rheology of food, cosmetics and pharmaceuticals. Curr Opin Colloid Interface Sci 4:288-293

Gallot T, Perge C, Grenard V, Fardin M-A, Taberlet N, Manneville S (2012). Ultrafast ultrasonic imaging coupled to rheometry: Principle and illustration. Rev Sci Instrum 84:045107

Geraud B, Bocquet L, Barentin C (2013) Confined flows of a polymer microgel. Eur. Phys. J. E 36:30

Germain D, Le Merrer M (2016) Bubbles slipping along a crenelated wall. EPL 115:64005

Ghosh S, van den Ende D, Mugele F, Duits MHG (2016) Apparent wall-slip of colloidal hard-sphere suspensions in microchannel flow. Colloid Surface A 491:50-56

Gibaud T, Barentin C, Manneville S (2008). Influence of boundary conditions on yielding in a soft glassy material. Phys Rev Lett 101:258302

Gibaud T, Taberlet N, Barentin C, Manneville S (2009) Shear-induced fragmentation of Laponite suspensions. Soft Matter 5:3026-3037

Gibaud T, Frelat D, Manneville S (2010) Heterogeneous yielding dynamics in a colloidal gel. Soft Matter 6:3482-3488.

Gibbs SJ, James KL, Hall LD, Haycock DE, Frith WJ, Ablett S (1996) Rheometry and detection of apparent wall slip for Poiseuille flow of polymer solutions and particulate dispersions by nuclear magnetic resonance velocimetry. J Rheol 40:425-440 
Gladden LF, Sederman AJ (2013) Recent advances in Flow MRI. J Magn Reson 229:2-11

Goddard JD (2003) Material instability in complex fluids. Annu Rev Fluid Mech 35:113-133

Golemanov K, Denkov ND, Tcholakova S, Vethamuthu M, Lips A (2008) Surfactant mixtures for control of bubble surface mobility in foam studies. Langmuir 24:9956-9961

Goyon J, Colin A, Ovarlez G, Ajdari A, Bocquet L (2008) Spatial cooperativity in soft glassy flows. Nature 454:84-87

Goyon J, Colin A, Ovarlez G, Ajdari A, Bocquet L (2010) How does a soft glassy material flow: finite size effects, non-local rheology, and flow cooperativity. Soft Matter 6:2668-2678.

Graham MD (1995) Wall slip and the nonlinear dynamics of large amplitude oscillatory shear flows. J Rheol 39:697-712

Granick S, Zhu Y, Lee H (2003) Slippery questions about complex fluids flowing past solids. Nat Mater 2:221227

Grenard V, Divoux T, Taberlet N, Manneville S (2014) Timescales in creep and yielding of attractive gels. Soft Matter 2014:1555-1571

Gulmus SA, Yilmazer U (2005) Effect of volume fraction and particle size on wall slip in flow of concentrated suspensions. J Appl Polym Sci 98:439-448.

Gulmus SA, Yilmazer U (2007) Effect of the surface roughness and construction material on wall slip in the flow of concentrated suspensions. J Appl Polym Sci 103:3341-3347.

Haavisto S, Salmela J, Jäsberg, Saarinen AT, Sorvari A, Karppinen A, Koponen A (2015) Rheological characterization of microfibrillated cellulose suspension using optical coherence tomography. TAPPI J 142:91304

Habibi M, Dinkgreve, Paredes J, Denn MM, Bonn D (2016) Normal stress measurements in foams and emulsions in the presence of slip. J Non Newtonian Fluid Mech 238: 33-43

Hartman Kok PJA, Kazarian SG, Briscoe BJ, Lawrence CJ (2001) Near-wall depletion in a flowing colloidal suspension. J Rheol 42:481-493

Hartman Kok PJA, Kazarian SG, Briscoe BJ, Lawrence CJ (2004) Effects of particle size on near-wall depletion in mono-dispersed colloidal suspensions. J Colloid Interface Sci 280:511-517

Hatzikiriakos SG, Dealy JM (1991) Wall slip of molten high density polyethylene. I. Sliding plate rheometer studies. J Rheol 35:497-523

Hatzikiriakos SG (2015) Slip mechanisms in complex fluid flows. Soft Matter 11, 7851-7856

Hollingsworth KG, Johns ML (2006) Rheo-nuclear magnetic resonance of emulsion systems J Rheol 48:787-803

Holmes WM, Callaghan PT, Vlassopoulos D, Roovers J (2004) Shear banding phenomena in ultrasoft colloidal glasses J Rheol 48: 1085-1102

Huang P, Guasto JS, Breuer KS (2006) Direct measurement of slip velocities using three-dimensional total internal reflection velocimetry. J Fluid Mech 566 :447-464

Hyun KH, Wilhelm M, Klein CO, Cho KS, Nam JG, Ahn KH, Lee SJ, Ewoldt RH, McKinley GK (2011) A review of nonlinear oscillatory shear tests: Analysis and application of large amplitude oscillatory shear (LAOS). Prog Polym Sci 36:1697-1753

Ianni F, Di Leonardo R, Gentilini S, Ruocco G (2007) Shear banding phenomena in a laponite suspension. Phys Rev E Stat Nonlin Soft Matter Phys 77:031406

Ireland PM, Jameson GJ (2009) Foam slip on surfaces of intermediate or low wettability. Chem Eng Sci 64:3859-3867 
Isa L, Besseling R, Poon WCK (2007) Shear zones and wall slip in the capillary flow of concentrated colloidal suspensions. Phys Rev Lett 98:198305

Jalaal M, Balmforth NJ, Stoeber B (2015) Slip of spreading viscoplastic droplets. Langmuir 31:12071-12075

Jana SC, Kapoor, B, Acrivos A (1995) Apparent wall slip velocity coefficients in concentrated suspensions of non-colloidal particles. J Rheol 39:1123-1132

Jaradat S, Harvey M, Waigh TA (2012) Shear-banding in polyacrylamide solutions revealed via optical coherence tomography velocimetry. Soft Matter 8:11677-1186

Jarny S, Roussel N, Rodts, S, Bertrand F, Le Roy R, Coussot P (2005) Rheological behavior of cement pastes from MRI velocimetry. Cem Concr Res 35:1873-1881

Jesinghausen S, Weiffen R, Schmid (2016) Direct measurements of wall slip and slip layer thickness of nonBrownian hard sphere suspensions in rectangular channel flows. Exp Fluids 57:153

Jofore BR, Erni P, Vleminckx G, Moldenaers P, Clasen C (2015) Rheology of microgels in single particle confinement. Rheol Acta 54:581-600

Jop P, Mansard V, Chaudhuri P, Bocquet L, Colin A (2012) Microscale rheology of a soft glassy material close to yielding. Phys Rev Lett 108:148301

Joseph DD (1997). Lubricated pipelining. Powder Technol 97:211-215

Joseph DD, Chen KP, Renardy YY(1997). Core annular flows. Ann Rev Fluid Mech 29: 65-90

Kalyon DM, Yaras P, Aral B, Yilmazer U (1993) Rheological behavior of a concentrated suspension: A solid rocket fuel stimulant. J Rheol 37:35-63

Kalyon DM, Lawal A, Yazici R, Yaras P, Railkar S (1999) Mathematical modeling and experimental studies of twin screw extrusion of filled polymers, Polym Eng Sci 39:1139-1151

Kalyon DM (2003) Comments on "A new method of processing capillary viscometry data in the presence of wall slip". J Rheol 47:1087-1088

Kalyon DM (2005) Apparent slip and viscoplasticity of concentrated suspensions. J. Rheol 49:621-640.

Kalyon DM (2010) An analytical model for steady coextrusion of viscoplastic fluids in thin slit dies with wall slip. Polym Eng Sci 50:652-664

Dhont JKG, Kang K, Kriegs H, Danko O, Marakis J, Vlassopoulos D (2017) Non-uniform flow in soft glasses of colloidal rods. Phys Rev Fluids 2 :043301

Kao SV, Nielsen LE, Hill CT (1975) Rheology of concentrated suspensions of spheres I. Effect of the liquidsolid interface. J Colloid Interface Sci 53:358-366

Keentok M, Milthorpe JF, O’Donovan E (1985). On the shearing zone around rotating vanes in plastic liquids: theory and experiment, J. Non-Newtonian Fluid Mech. 17:23-35

Khan SA, Schnepper CA, Armstrong RC (1988) Foam rheology: III. Measurement of shear flow properties. J Rheol 32:69-92

Klein CO, Hans W. Spiess HW, Calin A, Balan C, Wilhelm M (2007) Separation of the nonlinear oscillatory response into a superposition of linear, strain hardening, strain softening, and wall slip response. Macromolecules 40:4250-4259

Korhonen M, Mohtaschemi M, PuistoA, Illa X, Alava MJ (2015) Apparent wall slip in non-Brownian hardsphere suspensions. Eur Phys J E 38:46.

Kraynik A (1988) Foam flows. Ann Rev Fluid Mech 20: 325-357

Lam YC, Wang ZY, Chen X, Joshi SC (2007) Wall slip of concentrated suspension melts in capillary flows. Powder Technol 177:162-169 
Lauga E, Brenner M, Stone H (2007) Microfluidics: The no-slip boundary condition. Springer Handbook of Experimental Fluid Mechanics, 1219-1240 (Springer-Verlag Berlin Heidelberg).

Lawal A, Kalyon DM (1994a) Non-isothermal model of single screw extrusion of generalized newtonian fluids. Numer Heat Transfer 26:103-121

Lawal A, Kalyon DM (1994b) Single screw extrusion of viscoplastic fluids subject to different slip coefficients at screw and barrel surfaces. Polym Eng Sci 34:1471-1479

Lawal A, Kalyon DM (1998) Squeezing flow of viscoplastic fluids subject to wall slip. Polym Eng Sci 38:17931804

Leighton D, Acrivos A (1987) The shear-induced migration of particles in concentrated suspensions. J Fluid Mech 181:415-439

Le Merrer M, Lespiat R, Höhler R, Cohen-Addad S (2015). Linear and non-linear wall friction of wet foams. Soft Matter 11:368-381

Lester DR, Buscall R, Stickland AD, Scales PJ (2014) Wall adhesion and constitutive modeling of strong colloidal gels J Rheol 58:1247-1276

Lettinga P, Manneville S (2009) Competition between shear banding and wall slip in wormlike micelles. Phys Rev Lett 103:248302

Li Z, D’eramo L, Monti F, Vayssade A-L, Chollet B, Bresson B, Tran Y, Cloitre M, Tabeling P (2014). Slip length measurements using $\mu \mathrm{PIV}$ and TIRF-Based velocimetry. Isr J Chem 54:1589 - 1601

Li Z, D'eramo L, Lee C, Monti F, Yonger M, Tabeling P, Chollet B, Bresson B, Tran Y (2015) Near-wall nanovelocimetry based on total internal reflection fluorescence with continuous tracking. J Fluid Mech 766:147171

Lidon P, Villa L, Manneville S (2016) Power-law creep and residual stresses in a carbopol microgel. Rheol Acta DOI 10.1007/s00397-016-0961-4

Loppinet B, Dhont JKG, Lang P (2012) Near-field laser Doppler velocimetry measures near-wall velocities. Eur Phys J E 35: 62

Luu L-H, Forterre Y (2009) Drop impact of yield-stress fluids. J Fluid Mech 632:301-327

Ma L, Barbosa-Cánovas GV (1995) Rheological characterization of mayonnaise. Part I: Slippage at different oil and xanthan gum concentrations J Food Eng 25:397-408

Magnin A, Piau JM (1987) Shear rheometry of fluids with a yield stress. J Non-Newtonian Fluid Mech 23: 91106

Magnin A, Piau JM (1990) Cone-and-plate rheometry of yield stress fluids. Study of an aqueous gel. J NonNewtonian Fluid Mech 36:85-108.

Maillard M, Boujlel J, Coussot P (2015) Flow characteristics around a plate withdrawn from a bath of yield stress fluid. J Non-Newtonian Fluid 220:33-43

Mair RW, Callaghan PT (1997) Shear flow of wormlike micelles in pipe and cylindrical Couette geometries as studied by nuclear magnetic resonance microscopy. J Rheol 41:901-924

Mannheimer RJ (1972) Anomalous rheological characteristics of a high-internal-phase-ratio emulsion. J Colloid Interface Science 40:370: 382.

Manneville S, Bécu L, Colin A (2004) High-frequency ultrasonic speckle velocimetry in sheared complex fluids. Eur Phys J Appl Phys 28:361-373.

Manneville S, Colin A, Waton G, Schosseler F (2007) Wall slip, shear banding, and instability in the flow of a triblock copolymer micellar solution. Phys Rev E Stat Nonlin Soft Matter Phys 75:061502.

Manneville S (2008) Recent experimental probes of shear banding. Rheol Acta 47:301-318 
Mansard V, Colin A (2013) Local and non-local rheology of concentrated particles. Soft Matter 8:4025-4043

Mansard V, Bocquet L, Colin A (2014) Boundary conditions for soft glassy flows: slippage and surface fluidization, Soft Matter 10:6984-6989

Martoïa F, Perge C, Dumont PJJ, Orgéas L, Fardin, MA, Manneville S, Belgacem MN (2015) Heterogeneous flow kinematics of cellulose nanofibril suspensions under shear. Soft Matter 11:4742-4755

Marze S, Langevin D, Saint-Jalmes A (2008). Aqueous foam slip and shear regimes determined by rheometry and multiple light scattering. J Rheol 52:1091-1111

Mason TG, Bibette J., Weitz DA (1996). Yielding and flow of monodisperse emulsions. J Colloid Interface Sci $79: 439-448$

Meeker SP, Bonnecaze RT, Cloitre M (2004a) Slip and flow of soft particle pastes. Phys Rev Lett 92:198302

Meeker SP, Bonnecaze RT, Cloitre M (2004b) Slip and flow in pastes of soft particles: direct observation and rheology. J Rheol 48:1295-1320

Meeten GH, Sherwood JD (1992) Vane technique for shear-sensitive and wall-slipping fluids, in: P. Moldenaers, R. Keunings (Eds.), Proceedings of the 11th International Congress on Rheology: Theoretical and Applied Rheology, Elsevier, Amsterdam, 935-937.

Meeten GH (2004a) Squeeze flow of soft solids between rough surfaces. Rheol Acta 43:6-16

Meeten GH (2004b) Effect of plate roughness in squeeze flow rheometry. J Non-Newton Fluid 124:51-60

Métivier C, Rharbi Y, Magnin A, Bou Abboud A (2012) Stick-slip control of the Carbopol microgels on polymethyl methacrylate transparent smooth walls, Soft Matter 8:7365-7367

Meyer S, Richtering W (2005). Influence of polymerization conditions on the structure of temperature-sensitive poly (N-isopropylacrylamide) microgels. Macromolecules 38:1517-1519

Miller E, Rothstein JP (2007) Transient evolution of shear banding wormlike micellar solutions. J Non-Newton Fluid 143:22-37

Mooney M (1931) Explicit formulas for slip and fluidity. J Rheol 2, 210-222

Navier CLMH (1827) Sur les lois du mouvement des fluides. Mem Acad R Sci Inst Fr 6:389-440

Nazari B, Kumar V, Bousfield DW, Toivakka M (2016) Rheology of cellulose nanofibers suspensions: Boundary driven flow. J Rheol 60:1151-1159

Nechyporchuk O, Belgacem MN, Frédéric Pignon F (2014) Rheological properties of micro-/nanofibrillated cellulose suspensions: Wall-slip and shear banding phenomena. Carbohydr Polym 112:432-439

Nickerson C, Kornfield J (2005) A novel “cleat” geometry for suppressing wall slip. J Rheol 49: 865-874

Olmsted PD (2008). Perspectives on shear banding in complex fluids (2008). Rheol Acta 47:283-300

Ortega-Avila JB, Pérez-González J, Marín-Santibáñez BM, Rodríguez-González F, Aktas S, Malik M, Kalyon DM (2016) Axial annular flow of a viscoplastic microgel with wall slip. J Rheol 60:503-515

Ovarlez G, Cohen-Addad, Krishan K, Goyon J, Coussot P (2013) On the existence of a simple yield stress fluid behavior. J Non-Newton Fluid 193:68-79

Ozkan S, Gillece TW, Senak L, Moore DJ (2012) Characterization of yield stress and slip behaviour of skin/hair care gels using steady flow and LAOS measurements and their correlation with sensorial attributes. Int $\mathbf{J}$ Cosmetic Sci 34:193-201

Pal R (1998) Rheology of liquid membranes. Ind Eng Chem Res 37:2052-2058

Pal R (2000) Slippage during the flow of emulsion in rheometers. Colloid Surface A 162:55-66

Paredes J, Shahidzadeh N, Bonn D (2015) Wall slip and fluidity in emulsion flow. Phys Rev E Stat Nonlin Soft Matter Phys 92:042313

Patarin J, Galliard H, Magnin A, Goldschmidt B (2014) Vane and plateeplate rheometry of cheeses under oscillations and large strains: A comparative study and experimental conditions analysis. Int Dairy J 38:24-30. 
Persello J, A. Magnin A, Chang J, Piau JM, Cabane B (1994) Flow of colloidal aqueous silica dispersions. J Rheol 38:1845-1870

Pérez-González J, López-Durán JJ, Marín-Santibáñez BM, Rodríguez-González F (2012) Rheo-PIV of a yieldstress fluid in a capillary with slip at the wall. Rheol Acta 51:937-946.

Philippou M, Kountouriotis Z, Georgiou GC (2016) Viscoplastic flow development in tubes and channels with wall slip. J Non-Newton Fluid 234:69-81

Piau J M (2007) Carbopol gels: elastoviscoplastic and slippery glasses made of individual swollen sponges: meso- and macroscopic properties, constitutive equations and scaling laws. J Non-Newton Fluid 144:1-29

Pit R, Hervet H, Léger L (1999) Friction and slip of a simple liquid at a solid surface. Tribol Lett 7:147-152

Plucinski J, Gupta RK, Chakrabarti S (1998) Wall slip of mayonnaises in viscometers. Rheol Acta 37:256-269

Poumaere A, Moyers-González M, Castelain C, Burghelea T (2014) Unsteady laminar flows of a Carbopol gel in the presence of wall slip. J Non-Newton Fluid 205:28-40.

Princen H M (1985) Rheology of foams and highly concentrated emulsions. II. Experimental study of the yield stress and wall effects for concentrated oil-in-water emulsions. J. Colloid Interface Sci 105:150-171

Ragouilliaux A, Herzhaft B, Bertrand F, Coussot P (2006) Flow instability and shear localization in a drilling mud. Rheol Acta 46:261-271

Rabideau BD, Moucheront P, Bertrand F, Rodts S, Roussel N, Lanos C, Coussot P (2010) The extrusion of a model yield stress fluid imaged by MRI velocimetry. J Non-Newton Fluid $165: 394-408$

Raynaud JS, Moucheront P, Baudez JC, Bertrand F, Guilbaud JP, Coussot P (2002) Direct determination by nuclear magnetic resonance of the thixotropic and yielding behavior of suspensions. J Rheol 46, 709-732

Reimers MJ, Dealy JM (1996) Sliding plate rheometer studies of concentrated polystyrene solutions: Large amplitude oscillatory shear of a very high molecular weight polymer in diethyl phthalate. J Rheol 40:167-186

Roberts GP, Barnes HA (2001) New measurements of the flow-curves for Carbopol dispersions without slip artefacts. Rheol Acta 50:499-503

Rofe CJ, de Vargas L, Perez-González J, Lambert RK, Callaghan PT (1996) Nuclear magnetic resonance imaging of apparent slip effects in xanthan solutions. J Rheol 40, 1115-1128

Roman S, Merlo A, Duru P, Risso F, Lorthois S (2016) Going beyond $20 \mu \mathrm{m}$-sized channels for studying red blood cell phase separation in microfluidic bifurcations. Biomicrofluidics 10:034103.

Ross-Murphy SB (1995) Structure-property relationships in food biopolymer gels and solutions. J Rheol 39:1451-1463

Russell WB, Grant MC (2000) Distinguishing between dynamic yielding and wall slip in a weakly flocculated colloidal dispersion. Colloid Surface 161:271-282

Saïdi A, Martin C, Magnin A (2011) Effects of surface properties on the impact process of a yield stress fluid drop. Exp Fluids 51:211-224

Salmon J-B, Manneville S, Colin A, Pouligny B (2003a) An optical fiber based interferometer to measure velocity profiles in sheared complex fluids. Eur Phys J AP 22:143-154

Salmon J-B, Bécu L, Manneville S, Colin A (2003b) Towards local rheology of emulsions under Couette flow using Dynamic Light Scattering. Eur Phys J E 10:209-223

Sanchez-Reyes J, Archer LA (2003) Interfacial slip violations in polymer solutions: Role of microscale surface roughness. Langmuir 19:3304-3312

Saunders BR, Crowther HM, Vincent B (1997) Poly(methyl methacrylate)-co-(methacrylic acid) microgel particles: swelling control using pH, cononsolvency and osmotic deswelling. Macromolecules 30:482-487

Schall P, van Hecke M (2010) Shear Bands in Matter with Granularity. Annu Rev Fluid Mech 42:67-88

Schmatko T, Hervet H, Leger L (2005) Friction and slip at simple fluid-solid Interfaces: the roles of the molecular shape and the solid-liquid interaction. Phys Rev Lett 94:244501

Semwogerere D, Morris JF, Weeks ER (2007) Development of particle migration in pressure-driven flow of a Brownian suspension. J Fluid Mech 581:437-451. 
Seth J, Cloitre M, Bonnecaze RT (2008) Influence of short-range forces on wall-slip in microgel pastes. J Rheol $52: 1241-1268$

Seth JR, Mohan L, Locatelli-Champagne C, Cloitre M, Bonnecaze RT (2011) A micromechanical model to predict the flow of soft particle glasses. Nat Mater 10, 838-843

Seth J, Locatelli-Champagne C, Monti F, Bonnecaze RT, Cloitre M (2012) How do soft particle glasses yield and flow near solid surfaces. Soft Matter 8:140-148

Shewan HM, Stokes JS, Cloitre M (2017) Particle-wall tribology of slippery hydrogel particle suspensions. Soft Matter 13:2099-2106

Smay JE, Cesarano J III, Lewis JA (2002) Colloidal inks for directed assembly of 3-D periodic structures. Langmuir 18:5429-5437

Smith MI (2015) Fracture of jammed colloidal suspensions. Sci Rep 5:14175

Sochi T (2011) Slip at fluid-solid interface. Polym Rev 51:309-340

Solomon BR, Khalil KS, Varanasi KK (2014) Drag Reduction using Lubricant-Impregnated Surfaces in Viscous Laminar Flow. Langmuir 30:10970-0976

Soltani F, Yilmazer U (1998). Slip velocity and slip layer thickness in flow of concentrated suspensions. J Appl Polym Sci 70:515-522

Stickland AD, Kumar A, Kusuma TE, Scales PJ, Tindley A, Biggs S, Buscall R (2015) The effect of premature wall yield on creep testing of strongly flocculated suspensions. Rheol Acta 54:337-352

Stokes JR, Frith WJ (2008) Rheology of gelling and yielding soft matter systems. Soft Matter 4:1133-1140

Stokes JR, Boehm MW, Baier SK (2013) Oral processing, texture and mouthfeel: From rheology to tribology and beyond. Curr Opin Colloid Interface Sci 18:349-359

Tabuteau H, Baudez JC, Bertrand F., Coussot P (2004) Mechanical characteristics and origin of wall slip in pasty biosolids. Rheol Acta 43:168-174

Tang HS, Kalyon DM (2004) Estimation of the parameters of Herschel-Bulkley fluid under wall $\operatorname{slip}^{\wedge}$ using a combination of capillary and squeeze flow viscometers. Rheol Acta 43:80-88

Tang HS, Kalyon DM (2008) Time-dependent tube flow of compressible suspensions subject to pressure dependent wall slip: Ramifications on development of flow instabilities, J Rheol 52:1069-1090

Terriac E, Etrillard J, Cantat I (2006) Viscous force exerted on a foam at a solid boundary: Influence of the liquid fraction and of the bubble size. Europhys Lett 74:909-915

Tisné F, Doubliez L, Aloui F (2004) Determination of the slip layer thickness for a wet foam flow. Colloid Surface A 246:21-29

Tsitsilianis C, Iliopoulos I (2002) Telechelic polyelectrolytes in aqueous media. Macromolecules 2002:36623667

van Hecke M (2010) Jamming of soft particles: geometry, mechanics, scaling and isostaticity. J Phys: Condens Matter 22:033101

Vayssade A-L, Lee C, Terriac E, Monti F, Cloitre M, Tabeling P (2014) Dynamical role of slip heterogeneities in confined flows. Phys Rev E Stat Nonlin Soft Matter Phys 89:052309

Vermant J, Solomon MJ (2005) Flow-induced structure in colloidal suspensions. J Phys: Condens Matter 17:R187-R216

Vlassopoulos D, Cloitre M (2014) Tunable rheology of dense soft deformable colloids, suspensions. Curr Opin Colloid Interface Sci 19:561-574

Vlassopoulos D, Fytas G (2010) From polymers to colloids: engineering the dynamic properties of hairy particles. Adv Polym Sci 236:1-54

Walls HJ, Caines SB, Sanchez A M, Khan SA (2003) Yield stress and wall slip phenomena in colloidal silica gels. J Rheol 47:847-867

Wassenius H, Callaghan PT (2005) NMR velocimetry studies of the steady-shear rheology of a concentrated hard-sphere colloidal system. Eur. Phys. J. E 18:69-84 
Wein O, Tovchigrechko VV (1992) Rotational viscometry under presence of apparent wall slip. J Rheol 36:821 844

Westerberg LG, Lundström TS, Höglund E, Lugt PM (2010) Investigation of grease flow in a rectangular channel including wall slip effects using microparticle image velocimetry. Tribol T 53:600-609

Wilhelm M, Maring D, Spiess H-W (1998). Fourier-transform rheology. Rheol Acta, 37, 399-405

Wilson DI, Rough SL (2006) Exploiting the curious characteristics of dense solid-liquid pastes. Chem Eng Sci 61:4147-4154

Whitcomb PJ, Macosko CW (1978) Rheology of xanthan gum. J Rheol 22:493-505

Wolff K, Marenduzzo D, Cates ME (2012) Cytoplasmic streaming in plant cells: the role of wall slip. J R Soc Interface 9:1398-1408

Wyss H, Fernandez de Las Nieves A, Mattson J, Weitz DA (2011) Microgel Suspensions: Fundamentals and Applications (Wiley-VCH Verlag)

Yeow YL, Lee HL, Melvani AJ, Mifsud GC (2003) A new method of processing capillary viscometry data in the presence of wall slip. J Rheol 47:337-348

Yilmazer U, Kalyon DM (1989) Slip effects in capillary and parallel disk torsional flows of highly filled suspensions. J Rheol 33:1197-1212

Yoshimura A, Prud'homme RK (1988) Wall slip corrections for Couette and parallel disk viscometers. J Rheol 32:53-67

Zahirovic S, Lubansky A, Yeow YL, Boger, DV (2009) Obtaining the steady shear rheological properties and apparent wall slip data of a water-in-oil emulsion from gap-dependent parallel plate viscometry data. Rheol Acta 48:221-229

Zhu L, Sun N, Papadopoulos K, De Kee D (2001) A slotted plate device for measuring static yield stress. J Rheol 45:1105-1122

Xu C, Fie $\beta$ M, Willenbacher N (2017) Impact of wall slip on screen printing of front-side silver pastes for silicon solar cells. IEEE J Photovolt 7:129-135 